\title{
Peptide model helices in lipid membranes: insertion, positioning, and lipid response on aggregation studied by $\mathrm{X}$-ray scattering
}

\author{
Philipp E. Schneggenburger • André Beerlink • \\ Britta Weinhausen • Tim Salditt • Ulf Diederichsen
}

Received: 2 August 2010/Revised: 8 November 2010/Accepted: 12 November 2010/Published online: 23 December 2010

(C) The Author(s) 2010. This article is published with open access at Springerlink.com

\begin{abstract}
Studying membrane active peptides or protein fragments within the lipid bilayer environment is particularly challenging in the case of synthetically modified, labeled, artificial, or recently discovered native structures. For such samples the localization and orientation of the molecular species or probe within the lipid bilayer environment is the focus of research prior to an evaluation of their dynamic or mechanistic behavior. X-ray scattering is a powerful method to study peptide/lipid interactions in the fluid, fully hydrated state of a lipid bilayer. For one, the lipid response can be revealed by observing membrane thickening and thinning as well as packing in the membrane plane; at the same time, the distinct positions of peptide moieties within lipid membranes can be elucidated at resolutions of up to several angstroms by applying heavy-atom labeling techniques. In this study, we describe a generally applicable X-ray scattering approach that provides robust and quantitative information about peptide
\end{abstract}

Membrane-active peptides: 455th WE-Heraeus Seminar and AMP 2010 Workshop.

Electronic supplementary material The online version of this article (doi:10.1007/s00249-010-0645-4) contains supplementary material, which is available to authorized users.

\section{P. E. Schneggenburger $(\bowtie) \cdot$ U. Diederichsen}

Institut für Organische und Biomolekulare Chemie,

Georg-August-Universität Göttingen, Tammannstr. 2,

37077 Göttingen, Germany

e-mail: pschneg@gwdg.de

U. Diederichsen

e-mail: udieder@gwdg.de

A. Beerlink · B. Weinhausen - T. Salditt

Institut für Röntgenphysik, Georg-August-Universität Göttingen,

Friedrich-Hund-Platz 1, 37077 Göttingen, Germany

e-mail: tsaldit@gwdg.de insertion and localization as well as peptide/lipid interaction within highly oriented, hydrated multilamellar membrane stacks. To this end, we have studied an artificial, designed $\beta$-helical peptide motif in its homodimeric and hairpin variants adopting different states of oligomerization. These peptide lipid complexes were analyzed by grazing incidence diffraction (GID) to monitor changes in the lateral lipid packing and ordering. In addition, we have applied anomalous reflectivity using synchrotron radiation as well as in-house X-ray reflectivity in combination with iodine-labeling in order to determine the electron density distribution $\rho(z)$ along the membrane normal ( $z$ axis), and thereby reveal the hydrophobic mismatch situation as well as the position of certain amino acid side chains within the lipid bilayer. In the case of multiple labeling, the latter technique is not only applicable to demonstrate the peptide's reconstitution but also to generate evidence about the relative peptide orientation with respect to the lipid bilayer.

Keywords Peptide lipid interactions - Membrane active peptides - Model helices - X-ray scattering $\cdot$ Hydrophobic mismatch $\cdot$ Lipid chain correlation

$\begin{array}{ll}\text { Abbreviations } \\ \text { CD } & \text { Circular dichroism } \\ \text { DLPC } & \text { Dilauroyl-sn-glycero-3-phosphocholine } \\ \text { FRET } & \text { Förster resonance energy transfer } \\ \text { GID } & \text { Grazing incidence diffraction } \\ \text { P/L } & \text { Peptide/lipid } \\ \text { PNA } & \text { Peptide nucleic acid } \\ \text { RH } & \text { Relative humidity } \\ \text { RSM } & \text { Reciprocal space mapping } \\ \text { SPPS } & \text { Solid phase peptide synthesis } \\ \text { TM } & \text { Transmembrane }\end{array}$


TMD Transmembrane domain

TMH Transmembrane helix

\section{Introduction}

A quantitative, dynamic, and functional understanding of novel natural or synthetically modified peptide species in the native membrane environment requires information about the reconstitutability, positioning, and orientation with respect to the lipid bilayer matrix. Compared to protein complexes, membrane active peptides present relative structural simplicity and are frequently used to validate experimental approaches, techniques, and applications (Bechinger 2001; He et al. 1993). With the presented study, we are interested in evaluating the biophysical properties of a recently published motif of straightforward designed, aromatic, $\beta$-type polypeptides. This includes analysis of peptide positioning, orientation, and changes in bilayer thickness as well as the effect of peptide reconstitution on lateral lipid packing and ordering. Furthermore, we want to introduce a general and applicable X-ray-based approach that provides combined access to significant and quantitative parameters of peptide/lipid interaction on a molecular scale within the physiological membrane state. In this way, structural information, both in the bilayer plane and along the normal direction, can be determined.

While spectroscopic methods in their "standard" application, such as fluorescence emission (Bong et al. 2000; Krishnakumar and London 2007; Wimley and White 2000), circular dichroism (CD) (Chen and Wallace 1997; Koeppe and Andersen 1996), and transmission infrared (IR) spectroscopy (Küsel et al. 2007), produce qualitative information on the structure and localization of the peptide species, more advanced applications, such as oriented CD (Wu et al. 1990), attenuated total reflection (ATR) IR spectroscopy (Tamm and Tatulian 1997), Förster resonance energy transfer (FRET) experiments (parallax method) (Chattopadhyay and London 1987; Chung et al. 1992), solid state nuclear magnetic resonance (NMR) (Andronesi et al. 2004; Strandberg and Ulrich 2004), and electron paramagnetic resonance (EPR) techniques (Dzikovski et al. 2004; Marsh 1996), usually account for quantitative values in one dimension of the lipid bilayer without the need for complementary simulations or geometrical models (Naik and Krimm 1986a, b). Some of these techniques require membrane states or parameters that are far from physiologically or biologically relevant. In addition, sample preparation for some applications, e.g., frozen state samples for EPR spectroscopy, are often prone to uncertainties and artifacts.
Therefore, the sample preparation method itself has to be carefully evaluated, usually with significant effort.

It is obvious that no single technique can give complete structural information for each component of a fluid membrane system. However, in recent years the potential of X-ray scattering methods in probing peptide/lipid complexes, i.e., the membrane as well as the peptide structure, has been shown to be almost solely limited by the nonuniformity or patchiness of the peptide/lipid system itself (Qian et al. 2008; Salditt et al. 2006). Therefore, if samples of suitable quality can be obtained, the potential of a simultaneous analysis at the molecular scale of the incorporated peptide species, the surrounding lipid matrix, and the peptide/lipid interactions underlines the power of this analytical method. In addition, X-ray scattering is compatible with the fluid membrane state and capable of providing information for both dimensions of the lipid bilayer space.

\section{Applied methodologies}

The alignment of multilamellar membranes is quantified by the distribution function of the membrane normal vectors, which are directed along the $z$ axis perpendicular to the solid substrate on which the membranes are deposited. The central width of this distribution is called mosaicity. A narrow distribution or equivalently small mosaicity allows for a precise distinction between the scattering vector components, vertical $\left(q_{z}\right)$ and parallel $\left(q_{\|}\right)$to the lipid bilayer. Thereby, the multilamellar state of the samples, which can be almost fully hydrated by controlled relative humidity (Aeffner et al. 2009), provides a tremendous amplification of the scattering signal compared to single bilayer preparations. However, compared to single bilayer reflectivity, the multilamellar stacks usually impede the determination of the electron density $\rho(z)$ on an absolute scale, unless a full $q$-range fit of the electron density is performed (Constantin et al. 2003).

In principle, an ideal small-angle scattering experiment with respect to distribution, concentration, and structural integrity of the peptide species can yield multiple structural parameters (Fig. 1). The reflection geometry for aligned lipid films (Fig. 1i) is suited to determine the vertical density profile $\rho(z)$ of the bilayers, averaged in the $x y$ plane, e.g., by least-square fitting of the specular reflectivity curve (Constantin et al. 2003). The specular peaks are accompanied by diffuse scattering in the form of so-called Bragg sheets extending along $q_{\|}$. This scattering contribution can be assigned to lateral inhomogeneities at mesoscopic length scales (nanometers to a few micrometers) due to membrane fluctuations-always present in the fluid/ hydrated state-and peptide insertions causing local 


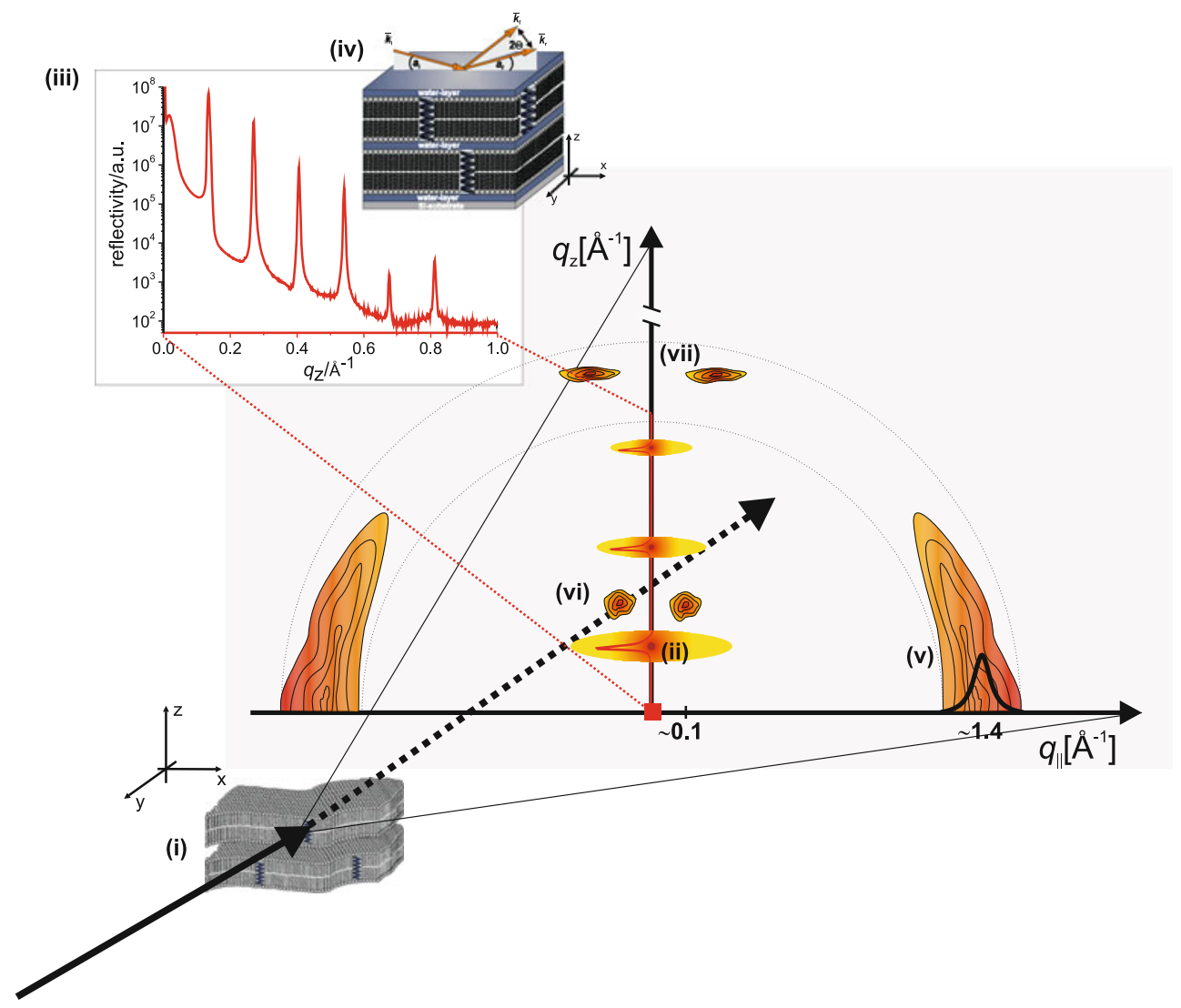

Fig. 1 Schematic representation of the reciprocal space for multilamellar membrane stacks $(i)$ as a function of the parallel $\left(q_{\|}\right)$and normal $\left(q_{z}\right)$ components of the momentum transfer $(q)$. The scheme shows the reflection geometry known as grazing incidence X-ray scattering (GISAXS) at small $q_{\|}$or grazing incidence diffraction (GID) at high $q_{\|}$. The recorded patterns include the diffuse Bragg sheets (ii) in the vicinity of the (specular) $q_{z}$ axis. In this study the $q_{z}$ components (iii) were measured by line scanning under specular

orientational disorders (Fig. 1ii), which break the lateral translation invariance.

While the vertical electron density distributions may also be obtained by fitting the diffuse Bragg sheet intensities of the reciprocal space for a single angle of incidence, we have used full scans with background correction (Fig. 1iii) in reflectivity mode (Fig. 1iv).

The lateral membrane structure at molecular length scales is accessible via the grazing incidence diffraction (GID) scattering geometry, at which the 2D detector (CCD camera) is moved out of the plane of incidence at grazing incidence and exit angles. The lipid chain correlation peak (Fig. 1v) is usually stretched around a circle with the radius $q=\left(q_{\|}^{2}+q_{z}^{2}\right)^{1 / 2} \cong 1.4 \AA^{-1}$ reflecting the distribution of acyl chain tilt angles with respect to the bilayer normal. The radial profile of the peak has a Lorentzian lineshape with a peak position corresponding to the inverse nearestneighbor distance of chains and its width reflecting the conditions (iv). At low $q_{z}$, the lipid acyl chain correlation maximum (v) is observed. Lorentzian fits yield the lateral lipid chain distance. The width of the acyl chain peak along $q_{\|}$gives information about the lipid ordering (correlation length); the angular width of the peak corresponds to the acyl chain tilt. In addition, superstructures (vi) and peptide geometries (helix maximum, vii) can be observed in some cases

lateral correlation length (see below). This peak is often broadened upon peptide insertion. A lateral correlation of membrane-inserted structures, e.g., a regular oligomerization as known from pore arrangements, can be detected and characterized from "superstructure peaks" (Constantin et al. 2007) in the small-angle region at low $q_{z}$ (Fig. 1vi). In addition, the conformation of the reconstituted peptide's structure can in some cases be deduced from the low $q_{\|}$ signal of molecular form factors, the so-called helix maximum (Fig. 1vii), or from helix peaks revealing intrahelical distances at high $q_{\|}$(not shown) as it has been revealed for the regularly organized and conformationally constrained alamethicin single helix (Spaar and Salditt 2003).

As we are interested in a general approach including a side-directed iodine-labeling for the reflectivity studies (Arbely et al. 2004), the present work focuses on data recording and analysis, as can be applied for any peptide/ lipid sample. 
Fig. 2 Molecular representation of the different D,L-alternating peptide types (a) ranging from the homodimeric species $\mathbf{1}$ to the designed hairpin 2 to the functionalized construct $\mathbf{3}$. Furthermore, the molecular structure of the homodimer 1 (b) (Küsel et al. 2007) as well as proposed equilibria (c) are sketched, either for homodimeric structures $(i)-(i i i)$ or the PNA-equipped transmembrane domains of $\mathbf{3}$ (v)-(vi) that undergo oligomerization driven by molecular recognition at the membrane's exterior

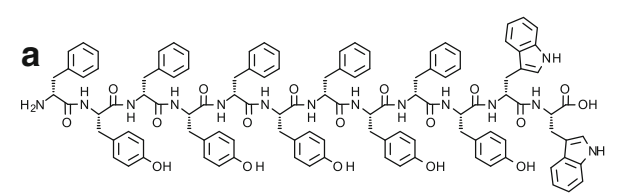

1

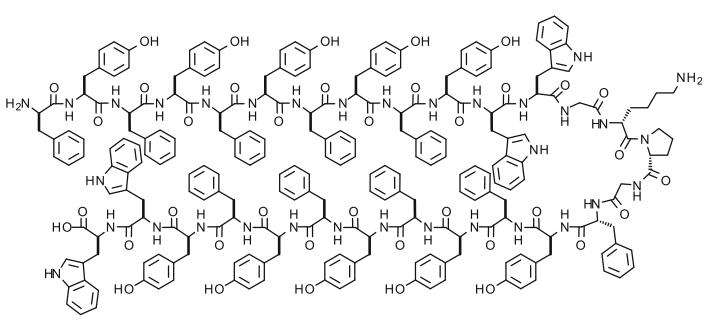

2

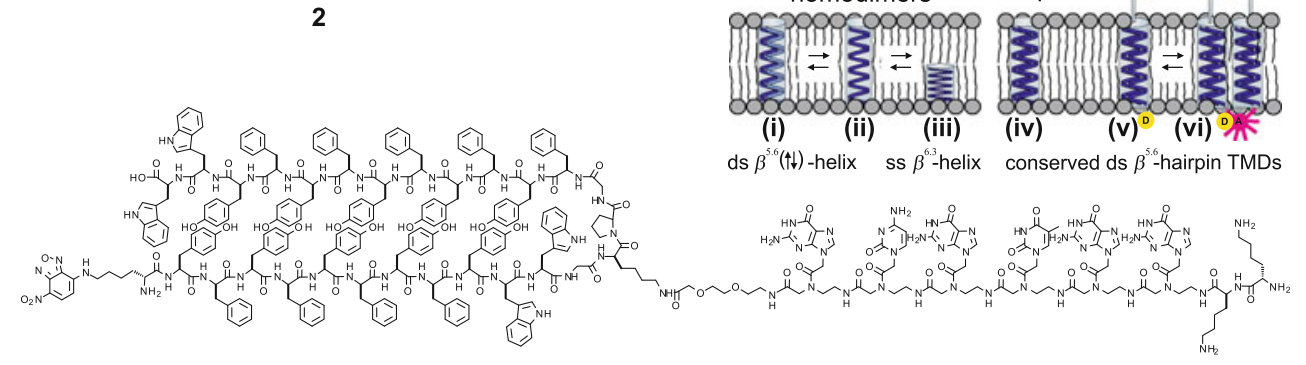

3
Design of the $\beta$-type peptide helix motif

In contrast to well established, commercially available peptide species, we concentrate our studies on a recently reported peptide model that was designed for spanning the membrane hydrophobic core and anchoring at the membrane/water interface. Therefore, this motif holds a tunable, conformationally stable peptide structure that is open for multiple functionalization (Schneggenburger et al. 2010). The D,L alternation of the peptide backbone concordant with a double-helical $\beta$-type shape is related to natural peptide antibiotics such as gramicidin A (Kelkar and Chattopadhyay 2007) or feglymycin (Bunkóczi et al. 2005). In addition, the studied sequences are unique on their own due to their almost purely aromatic side-chain composition (Alexopoulos et al. 2004; Küsel et al. 2007). On the basis of the crystallographically elucidated watersoluble nonameric sequence $\mathrm{H}$-(Tyr-Tyr) ${ }_{4}-\mathrm{Lys}-\mathrm{OH}$ (note: underlined amino acids indicate the $\mathrm{D}$-conformer), the homodimeric dodecamer H-( $\underline{\text { Phe-Tyr }})_{5}$-Trp-Trp-OH (1) (Fig. 2a, b) and the covalently linked hairpin species H-(Phe-Tyr) ${ }_{5}$-Trp-Trp-Gly-Lys-Pro-Gly-(Phe-Tyr) ${ }_{5}$ Trp-Trp-

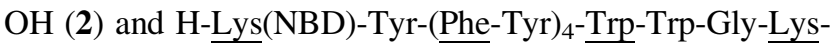
$\left(\left[\mathrm{C}_{2} \mathrm{H}_{4} \mathrm{O}\right]_{2}-\mathrm{CH}_{2}-\mathrm{CO} \text {-gcgtgg-Lys-Lys)-Pro-Gly-(Phe-Tyr }\right)_{5-}^{-}$ Trp-Trp-OH (3) (Fig. 2a) (note: gcgtgg represents the nucleobase sequence of aminoethylglycine peptide nucleic acid monomers) were derived via synthetic modification applying solid-phase peptide synthesis (SPPS) and loop design (Schneggenburger et al. 2010). The transmembrane helices of the latter structures (1-3) are well adapted to the membrane environment as optimized by sequence variation in length and composition (Küsel et al. 2007).

Through the execution of design studies of the peptide substrates, the preservation of the $\beta^{5.6}$ helical structure (Fig. 2b; the superscript denotes the periodicity of the helix), as found for the homodimeric species $\mathbf{1}$, was maintained for the final hairpin prototype $\mathbf{2}$ via constant comparison of the respective circular dichroism data resulting from several tested constructs (Schneggenburger 2010).

As there has been strong evidence for a conformational and monomer-dimer equilibrium of the homodimeric structure 1 involving antiparallel oriented $\beta^{5.6}$ double and $\beta^{6.3}$ single helices (Fig. 2c, i-iii) (Küsel et al. 2007), a covalent linkage of both strands seemed to be mandatory in order to circumvent the described equilibria. This goal was fulfilled through the synthesis of the peptide hairpin 2 (Fig. 2c, iv) allowing for further studies that address peptide oligomerization within lipid model membrane complexes. The helix assembly is, thereby, driven by molecular recognition of the peptide nucleic acid (PNA) moieties at the membrane's exterior (Fig. 2c, v-vi; Schneggenburger 2010). The attachment of fluorescence probes and peptide nucleic acid (PNA) recognition moieties led to functional constructs, such as compound $\mathbf{3}$, that have already been tested for their dynamic dimerization within lipid bilayer structures via Förster resonance energy transfer (FRET) assays (Schneggenburger et al. 2010). 
Here, we probe the membrane reconstitution of the three presented kinds of $\beta$-helical species via GID and reciprocal space mapping (RSM) and evaluate their influence on the lateral lipid packing by addressing the lipid chain correlation peak. In the respective studies, parameters such as the sample composition, the relative humidity $(\mathrm{RH})$, and the peptide-to-lipid (P/L) ratio are varied. The obtained results can be explained by a qualitative model that predominantly takes into account a hydrophobic mismatch situation as well as a lateral packing and ordering of the annular lipid shell that is correlated to the extent of peptide-lipid contact surface. Furthermore, we analyze the differentially (double) iodine-labeled hairpin species of type $\mathbf{2}$ via anomalous and in-house reflectivity elucidating their positioning with respect to the membrane normal $(z$ axis) and confirming their transmembrane orientation. These experiments created the basis for a functional FRET assay that was reported elsewhere (Schneggenburger et al. 2010).

\section{Materials and methods}

\section{Sample preparation and environment}

Solid supported stacks of typically 1,000 aligned DLPC bilayers with a cholesterol ( $\mathrm{Chol}$ ) content of $5 \%$ and differing amounts of the respective peptide species were prepared from stock solutions following procedures described in the literature (Seul and Sammon 1990). Polished and cleaned (sonication in $\mathrm{MeOH}$ and ultrapure water, 15 min each) Si wafers with $\langle 100\rangle$ orientation and a thickness of $625 \mu \mathrm{m}$ (Silchem, Freiberg, Germany) were used as substrates. Stock solutions of DLPC and Chol in chloroform were prepared at concentrations of 40 and $3 \mathrm{mg} / \mathrm{ml}$, respectively. Peptide stocks were composed of $\mathrm{MeOH} / \mathrm{DCM} / \mathrm{EtOH} 4 / 3 / 3$ (v/v/v) at $6 \mathrm{mg} / \mathrm{ml}$ concentration. For $\mathrm{P} / \mathrm{L}$ ratios between $1 / 10$ and $1 / 50$ in a definite volume of 80 or $150 \mu \mathrm{l}$, mixtures of stock solutions were spread onto cleaned, horizontally mounted silicon substrates with dimensions of $10 \times 15 \mathrm{~mm}(80 \mu \mathrm{l}$, beamline experiments) or $15 \times 25 \mathrm{~mm}(150 \mu \mathrm{l}$, in-house experiments), respectively. The coated Si wafers were covered with a watch glass, and the solvent was carefully evaporated in a flowbox overnight to prevent film rupture and fast dewetting. Subsequently, reduced pressure was applied for an additional $12 \mathrm{~h}$. The resulting film-covered substrates were stored at $4^{\circ} \mathrm{C}$ until use.

For measurements, the prewarmed $\left(40^{\circ} \mathrm{C}, 1 \mathrm{~h}\right)$ and rehydrated (saturated water vapor atmosphere) samples were placed in home-built sample cells with Teflon sealing and either PE foil or kapton windows. A setup was applied at which the RH could be adjusted by PID control, remaining stable within $0.1 \%$ (Aeffner et al. 2009). The temperature $(\mathrm{T})$ of the sample chamber, the water reservoir, and the pipings was controlled as well. The RH/T sensors and mass flow controllers were interfaced with the diffractometer controls (in-house and beamline), enabling the usage of long and fully automated scan macros including RH and $\mathrm{T}$ as parameters. Unless otherwise stated, GID experiments were carried out at $\mathrm{RH}=94 \%$, and the reflectivity experiments were performed at $\mathrm{RH}=90 \%$, both at $20^{\circ} \mathrm{C}$. The chambers were mounted to the respective goniometers with the sample oriented either horizontally (beamline) or vertically (in-house) depending on the diffractometer setup. The X-ray beam enters and exits the chamber through kapton (in-house) or PE foil (beamline) windows.

\section{Experiments}

GID and anomalous reflectivity experiments were performed with the insertion device 01 (ID01) undulator beamline at the European Synchrotron Radiation Facility (ESRF, Grenoble, France), while in-house reflectivity measurements were carried out with a home-built diffractometer.

\section{X-ray scattering: GID (IDO1, ESRF)}

In GID mode the sample was tilted at a fixed angle of incidence close to the critical angle of total external reflection $\alpha_{c}$ in order to optimize the lateral scattering intensity and to minimize background scattering caused by the substrate. The vertical scattering depth along $z$ is, thereby, tuned by the angle of beam incidence $\alpha_{i}$ and the angle of the scattered beam $\alpha_{\mathrm{f}}$ (compare to Fig. 3). GID experiments were performed at $17 \mathrm{keV}$ radiation using a Peltier cooled 2D CCD detector (Princeton Scientific Instruments, Princeton, NJ, USA) providing a resolution of $1,340 \times 1,300$ pixels (pixel size: $48 \times 49 \mu \mathrm{m}^{2}$; active area: $64.3 \times 63.7 \mathrm{~mm}^{2}$ ) that was mounted at approximately $19.5 \mathrm{~cm}$ from the sample. The beam was cut by the entrance slits to $0.7 \times 0.7 \mathrm{~mm}^{2}$ in front of the sample; no beam restrictions were set on the detector side. Three different types of measurements were performed:

1. After placing the sample roughly horizontally and adjusting the specular axis to the center of the CCD, four images were taken along the $q_{z}$ direction under stepwise increase of the angle of beam incidence $\left(\Delta \alpha_{i}=0.45^{\circ}\right)$ enabling the calculation of the actual $\alpha_{i}$ as well as the exact sample detector distance and allowing for scanning the low $q_{\|}$space for peaks resulting from superstructures (Fig. 3).

2. Adjustment of the specular axis at one side of the CCD and coverage by a lead stripe facilitated scanning the 


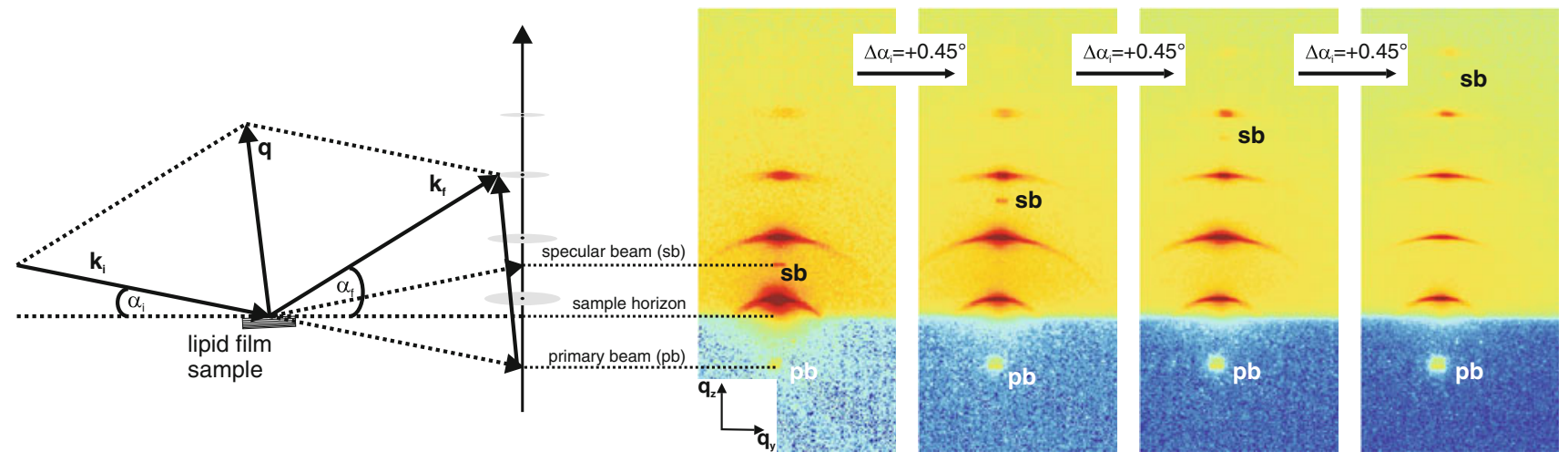

Fig. 3 GID scattering geometry (left) and exemplary results from low $q_{\|}$scanning at different angles of $\alpha_{\mathrm{i}}$ in search of "superstructure peaks" (right) also required for determination of parameters concerning the scattering geometry. As the angle of incidence increases, the specular beam reflex $(s b)$ moves along the $q_{z}$ axis. The primary beam $(p b)$ is located at the origin of the $q_{z}$ axis, here shadowed by the sample horizon

Salditt 2007). Therefore, no automated absorbers could be used during reflectivity or offset scans; absorbers had to be calibrated in advance of data collection. A monitor, mounted directly in front of the sample, was used to control the beam intensity. To further reduce dose and avoid beam damage, the reflectivity curves and offset scans were recorded only in the vicinity of the Bragg reflections at calculated positions.

In resonant reflectivity experiments, the scattering power of iodine labels was used to retrieve selective structural information of the iodine positioning with respect to the membrane normal by varying the incidence of the X-ray radiation close to the iodine $L_{\mathrm{III}}$ absorption edge around $E=4.5575 \mathrm{keV}$ (Gullikson 1995-2008). The scattering length becomes explicitly dependent on the $X$-ray energy $E$ near the absorption edge, according to $f=f_{0}+f^{\prime}(\mathrm{E})+i f^{\prime \prime}(\mathrm{E})$, where $f_{0}$ is the number of electrons of the ion (nonresonant term) and $f^{\prime}$ and $i f^{\prime \prime}$ are the real and imaginary corrections of the scattering factor, respectively (Evans and Pettifer 2001). Both $f^{\prime}$ and $f^{\prime \prime}$, connected by the Kramers-Kronig relations, strongly vary in the vicinity of an absorption edge and can be measured $\left(f^{\prime \prime}\right)$ or obtained from databases. At an absorption edge the absolute values of $f^{\prime}$ and $f^{\prime \prime}$ are the highest with respect to nonresonant energy regions, where $f \approx f_{0}$. Thus, in a resonant diffraction experiment it is very important to determine the energy value of the absorption edge accurately to a resolution of about $1 \mathrm{eV}$. It is known that the scattering factors $f^{\prime}$ and $f^{\prime \prime}$ of a chemical species within a sample, here iodine, depend very sensitively on its environment. Since there are many influences on these values, their accurate calculation by theoretical consideration is not feasible. Therefore, it is the most reliable strategy to measure $f^{\prime \prime}$ and calculate $f^{\prime}$ during the experimental setup (Als-Nielsen and McMorow 2001). Experimentally, it is possible to access $f^{\prime \prime}$ by absorption or fluorescence measurements. In order to 
mimic the hydrophobic environment within a lipid membrane where peptides should be placed, absorption measurements were performed using quartz capillaries (700 $\mu \mathrm{m}$ diameter) filled with $0.5 \mathrm{M}$ iodoform dissolved in chloroform. Instead of measuring $f^{\prime \prime}$ and calculating $f^{\prime}$ the distinct iodine $L_{\mathrm{III}}$ edge was found by varying the beam energy about $\pm 0.025 \mathrm{keV}$ around the tabulated $L_{\mathrm{III}}$ edge by means of modulating the undulator gap and detecting the absorption. The iodine $L_{\mathrm{III}}$ edge was found at $E=4.5578 \mathrm{keV}$. For the contrast variation in the anomalous scattering experiments, the reflectivity measurements were performed at energies of 4.5578 and $5.8000 \mathrm{keV}$.

\section{In-house X-ray reflectivity (nonisomorphic samples)}

For comparison and because of higher resolution (less absorption at higher energies) and enhanced contrast variation [see tabulated atomic scattering factor $f^{\prime}$ of iodine (Gullikson 1995-2008)], in-house reflectivity experiments have been performed with the same sample composition as applied in synchrotron experiments. As the beam energy cannot be varied at in-house experimental stations, nonisomorphic samples, with respect to the iodine labels, were used for the home-built diffractometer. The in-house experimental station is a stationary $\Theta / 2 \Theta$ diffractometer with a Seifert long fine focus $\mathrm{X}$-ray tube holding a $\mathrm{Cu}$ anode $(U=35 \mathrm{kV}, I=40 \mathrm{~mA})$ and a Cyberstar point detector. The X-ray beam is monochromatized and parallelized by a Göbel mirror selecting the $\mathrm{Cu}-\mathrm{K}_{\alpha}$ line $(E=8.048 \mathrm{keV}, \lambda=1.541 \AA)$. The primary beam intensity is on the order of $10^{9}$ counts per second (cps). Automatized absorbers are used to avoid detector saturation for $2 \Theta$ close to zero and at first Bragg reflexes.

The sample is mounted to a Huber goniometer for which three linear stages for $x, y$, and $z$ translation are used to place the sample in the center of rotation. The sample-todetector distance is $400 \mathrm{~mm}$. The lateral dimensions of the primary beam $\left(1 \times 5 \mathrm{~mm}^{2}\right)$ are defined by two slits in the horizontal and the vertical direction (S1). A vertical slit behind the sample (S2) screens scattering that does not stem from the sample; additional slits in front of the detector (S3) define the resolution of the instrument. All scans are performed at slit widths of $2 \mathrm{~mm}$ (S2) and $0.5 \mathrm{~mm}$ (S3). For the given sample-detector distance, the latter yields a resolution of $0.07^{\circ}$ or $0.01 \AA^{-1}$. With these settings, the profile of the primary beam typically has a full width at half maximum (FWHM) of $0.2^{\circ}$. The diffractometer motor and the detector controls as well as the monitor counter readout are accomplished by the SPEC software (Certified Scientific Software, Cambridge, MA, USA).
Analysis

\section{$2 D$ data}

Two-dimensional resolved maps of the $q$ space were derived from the CCD images via data treatment that includes (1) positioning of the beam center, (2) polarization correction, (3) elimination of dead pixels, and (4) scaling. Data treatment was performed by applying a self-written MATLAB (The MathWorks, Natick, MA, USA) tool (Weinhausen 2010). This tool further allows for sectioning through the reciprocal space at a definite angle $(\phi)$ between $q_{z}$ and $q_{\|}$leading to extraction of the intensity courses along these sections via averaging over an angular ROI five pixels wide. The respective intensity profiles were linearized. Lorentzians with linear backgrounds were fitted to the extracted functions:

$I(q)=I_{0} \frac{\omega^{2}}{\left(q-q_{0}\right)^{2}+\omega^{2}}+m q+b$

where $\omega$ is the half width at half maximum, $q_{0}$ is the peak center, $I_{0}$ is the maximum of the Lorentzian without the linear offset, $m$ is the slope of the linear background, and $b$ is the constant offset of the Lorentzian. The correlation length $\xi=1 / \omega$ and the average chain distance in real space were calculated:

$a \cong \frac{9 \pi}{4 q_{0}}-\frac{3 \omega}{2 q_{0}}$.

$1 D$ data

For analysis of the resulting reflectivities, a self-written MATLAB software tool was applied (Reusch 2009). The reflectivity data were plotted as a function of the vertical momentum transfer $q_{z}$ after subtraction of the diffuse scattering (offset scan) and illumination correction. The electron density profiles were calculated by applying an empirical Fourier Synthesis (FS) scheme, exploiting the area under Bragg peak intensities $I_{n}$, as it is used for such multilamellar lipid membranes (Münster et al. 2000; Spaar et al. 2004; Wu et al. 1995). In simple terms the onedimensional electron density profile $\rho(z)$ was obtained via Fourier synthesis method from the integrated peak intensities via Gaussian fitting, applying the Lorentz correction factor $1 / q_{z}^{2}$ and phases,,,,,,--+---- in accordance with the number of observed Bragg reflexes. The phases $v_{n}$ are reduced to positive/negative signs due to the point symmetry and were reconstructed in accordance with the 1D swelling method approach using Eq. 3 to obtain the continuous form factor $F\left(q_{z}\right)$ with its relative amplitude $\left|F\left(q_{z, n}\right)\right|$ and phase $v_{n}$ : 
$F\left(q_{z}\right)=\sum_{n=1}^{n=n_{\max }} v_{n}\left|F\left(q_{z, n}\right)\right| \sin c\left(\frac{d}{2}\left(q_{z}-q_{n, z}\right)\right)$.

The scattering vectors are represented by $q_{z}$ and $q_{z, n}$, and $d$ is the membrane periodicity (Aeffner et al. 2009). The phases have been extracted by the 1D swelling method of pure DLPC-lipid samples (Schneggenburger et al. 2009). The electron density profile $\rho(z)$ normal to the interface is computed by $N_{0}$ Fourier coefficients $f_{n}=I_{n} q_{z}$ (Eq. 4):

$\rho(z)=\sum_{n=1}^{N_{0}} v_{n} n \sqrt{I_{n}} \cos \left(\frac{2 \pi n z}{d}\right)$.

The factor $n$ in front of the Bragg peak intensity $I_{n}$ follows from an empirical correction factor to calculate the $n$th Fourier coefficient. The curves have been normalized by scaling higher-order Bragg peaks to the area under the first Bragg peak. General aspects of reflectivity experiments are discussed elsewhere (Salditt et al. 2002).

\section{Results and discussion}

Insertion and lateral lipid response by reciprocal space mapping

The homodimeric representative of the applied peptide motif $\mathrm{H}$-(Tyr-Tyr $)_{4}-\mathrm{Lys}-\mathrm{OH}$ has already been shown to enable intermolecular interaction via its phenolic sidechain pattern within the aqueous phase (Alexopoulos et al. 2004). The homodimer species 2 adopts a membrane spanning orientation in DLPC bilayers (Küsel et al. 2007) and likewise tends to aggregation (Schneggenburger et al. 2009). Therefore, the GID approach was undertaken to evaluate if the latter observation of a membrane insertion and transmembrane alignment is likewise true for the designed hairpin species (2) and the recognition system (3) (Fig. 2). Furthermore, it was our aim to screen the samples for any evidence of lateral peptide interaction, i.e., the formation of higher-order structures (low $q_{\|}, q_{z}$ ), as sometimes indicated by superstructure peaks (Constantin et al. 2007) as well as for regular intrahelical distances that can be revealed from the occurence of so-called helix peaks (Spaar et al. 2004).

\section{Samples and parameters of GID experiments}

Highly aligned membrane stacks of either a pure lipid matrix (DLPC/Chol) or differentially composed peptide/ lipid compositions (Fig. 2) were analyzed. The lipid phase of each sample contained 5\% cholesterol to enhance the surface mosaicity and reduce bilayer fluctuations allowing for pronounced Bragg reflections and correspondingly higher resolution in the reflectivity experiments (Chen and Rand 1997; Mouritsen and Zuckermann 2004). The particular sample composition, the variation of parameters, and the obtained data are shown in Table 1.

The direct comparison between undisturbed DLPC/Chol membranes and membranes with either homodimeric or hairpin structures was carried out via analysis of samples 1-3 (Table 1). The homodimer 4 [=H-(Phe(4I)-Tyr-(PheTyr) $)_{4}$-Trp-Trp-OH] was embedded at a $\overline{\mathrm{P} / \mathrm{L}}$ ratio of $\overline{1 / 10}$ while the hairpin system 5 [=H-(Phe-Tyr) 3 -Phe(4I)-TyrPhe-Tyr-Trp-Trp-Gly-Lys-Pro-Gly-(Phe-Tyr) $)_{2}$-Phe(4I)-(TyrPhe $)_{2}$-Tyr-Trp-Trp-OH] was embedded at $\mathrm{P} / \mathrm{L}=1 / 20$, both leading to a peptide-to-helix ratio of $1 / 20$. The equimolar mixture of species 3 and $\mathbf{6}$ [= H-Lys(TAMRA)-Tyr-(PheTyr $)_{4}$-Trp-Trp-Gly-Lys- $\left(\left[\mathrm{C}_{2} \mathrm{H}_{4} \mathrm{O}\right]_{2}-\mathrm{CH}_{2}\right.$-CO-gcgtgg-LysLys)-Pro-Gly-(Phe-Tyr $)_{5}$-Trp-Trp-OH] representing the recognition system could at least be incorporated into multilamellar DLPC stacks at $\mathrm{P} / \mathrm{L}=1 / 40$ avoiding precipitation. A second hairpin sample (sample 4) including compound 5 was prepared at a $\mathrm{P} / \mathrm{L}$ ratio of $1 / 40$. Therefore, samples 3 and 4 were analyzed to account for the concentration dependency of the observed lipid response. In order to evaluate the applied methodology and due to the fact that the recognition system (3/6) should be most amenable to changes in RH because of its polar PNA moieties facing the water layers, a series of experiments was performed at different RHs ranging from $94 \%$ to $25 \%$. All other samples were analyzed at $\mathrm{RH}=94 \%$.

In searching for potential superstructure peaks in the low $q_{z}$ range and the helix maximum at higher $q_{z}$, first, scans of the small $q_{\|}$space along the $q_{z}$ axis were performed in GID mode under variation of the angle of beam incidence (Fig. 3). These scans were followed by addressing the far $q_{\|}$space under attenuation of the specular axis, yielding a combination of exposures that provide a partial overlap (data not shown).

As neither reflections resulting from superstructures nor from helix peaks could be observed for the reconstituted peptide species 3-6, quantitative images (accumulations) of the $q$ space were taken, focusing on the lipid chain correlation peak (cc peak). In analyzing the lateral lipid bilayer response of different samples upon peptide insertion, the cc peak gives indirect information on the peptide reconstitution and peptide/lipid interactions. Attenuation of the specular axis at low angles of incidence, e.g., $\alpha_{\mathrm{i}} \approx 0.4^{\circ}$, avoiding overexposure of the detector provided sufficient access to the cc peak at low $q_{z}$ (Fig. 4a). Taking images of the reciprocal space at higher $\alpha_{\mathrm{i}}$ (e.g., $\alpha_{\mathrm{i}} \approx 2.5^{\circ}$ ) without attenuation led to unacceptably high sample horizons (shadowed low $q_{z}$ ) that were not applicable for analysis of the cc peak.

Corrections of the scattering distribution (dark image, polarization), normalization (monitor signal, counting 
Table 1 Composition of the different peptide/lipid samples addressed by grazing incidence diffraction (GID)

\begin{tabular}{|c|c|c|c|c|c|c|}
\hline Sample no. & Compounds & Species type & $\mathrm{P} / \mathrm{L}$ ratio & $\mathrm{RH}(\%)$ & $a(\AA)$ & $\xi(\AA)$ \\
\hline 1 & Pure lipid sample & - & - & 94 & $4.948 \pm 0.012$ & $4.10 \pm 0.24$ \\
\hline 2 & $H-E(4)\}-Y-\left(E-Y_{4}-\underline{W}-W_{-O H}(4)\right.$ & HD & $1 / 10(\equiv 1 / 20$ helices $)$ & 94 & $4.896 \pm 0.009$ & $5.71 \pm 0.21$ \\
\hline 3 & \multirow{2}{*}{ 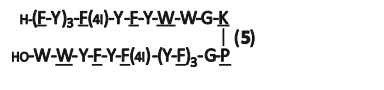 } & \multirow[t]{2}{*}{$\mathrm{HP}$} & $1 / 20$ & 94 & $4.886 \pm 0.005$ & $5.44 \pm 0.11$ \\
\hline 4 & & & $1 / 40$ & 94 & $4.937 \pm 0.005$ & $5.94 \pm 0.13$ \\
\hline 5 & \multirow{3}{*}{ 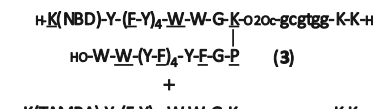 } & \multirow{5}{*}{$\begin{array}{l}\text { Functionalized HPs } \\
\text { (recognition system) }\end{array}$} & $1 / 40$ & 94 & $4.925 \pm 0.004$ & $6.31 \pm 0.12$ \\
\hline 6 & & & $1 / 40$ & 80 & $4.908 \pm 0.004$ & $6.78 \pm 0.13$ \\
\hline 7 & & & $1 / 40$ & 60 & $4.886 \pm 0.002$ & $7.19 \pm 0.09$ \\
\hline 8 & 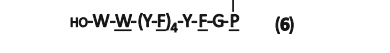 & & $1 / 40$ & 40 & $4.860 \pm 0.003$ & $7.18 \pm 0.07$ \\
\hline 9 & & & $1 / 40$ & 25 & $4.828 \pm 0.002$ & $7.02 \pm 0.08$ \\
\hline
\end{tabular}

In addition to the peptide component, the $\mathrm{P} / \mathrm{L}$ ratio and the relative humidity (RH) were likewise varied. For all samples, i.e., the pure lipid phase (sample 1) as well as the varied P/L compositions (samples 2-9), the lipid fraction consists of DLPC/Chol (95/5). The values of the lipid acyl chain distance $a$ and the lipid chain correlation length $\xi$ were obtained via analysis of the lipid chain correlation peak by Lorentzian fitting of the intensity traces at $\phi=8^{\circ}$

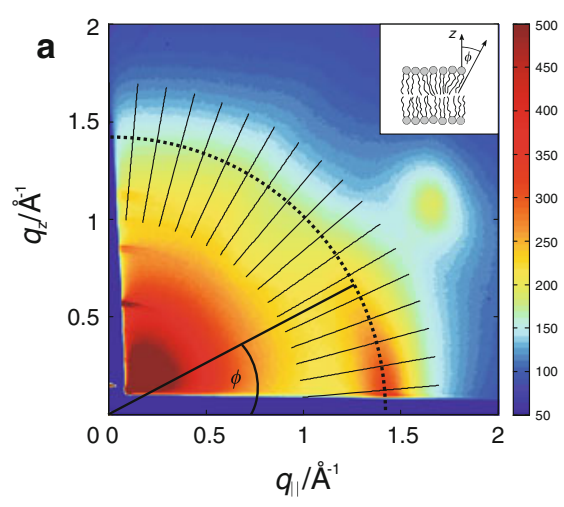

Fig. 4 An example of reciprocal space mapping (a) is shown for sample 9 (Table 1). The primary beam is located in the origin of the coordinate system with the specular axis covered by a lead stripe on the left hand side. Extensions of the Bragg sheets can be identified as streaks. The lipid chain correlation peak (cc peak) stretches around a

time), and coordinate transformation to $\left(q_{\|}, q_{z}\right)$ coordinates based on the calculated sample-to-detector distance led to the 2D intensity distribution in the reciprocal space (Fig. 4a). Intensity profiles (Fig. 4b) of the cc peak were extracted by interpolation along radial sections through the reciprocal space (black lines in Fig. 4a) for different angles $\phi$ corresponding to the tilting of lipid acyl chains in real space (Fig. 4a, inset). The root mean square deviation of the intensity was estimated via extraction of five neighboring profiles within a distance of $\Delta \phi=0.2^{\circ}$ (1-2 pixels). A Lorentzian function (Eq. 1) was fitted to the intensity traces via a nonlinear least squares method using a trust region.

The Lorentzian function fits the intensity profiles $[I(\phi)]$ very well for all $\phi$. For calculation of the average lipid acyl chain distance $a$ (Eq. 2) and the correlation length $\xi$ only intensity traces at small $\phi$ were used in order to minimize

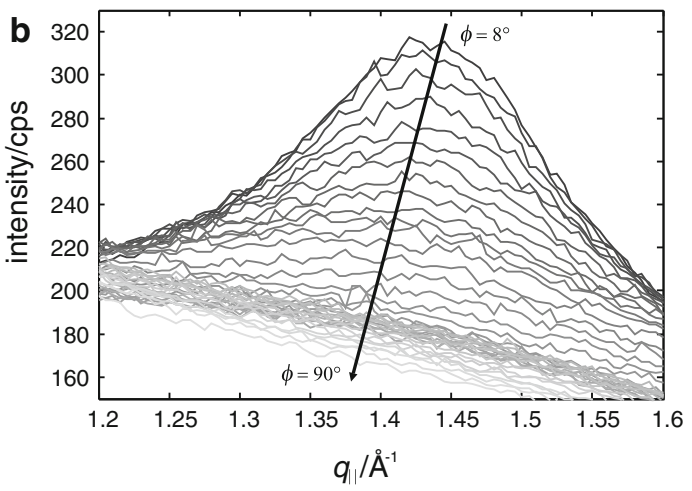

circle with the radius $q \cong 1.4 \AA$ (dotted line). The peak at $q_{\|} \approx 1.7$, $q_{z} \approx 1.1$ results from the silicon substrate and can be applied for alignment. From sections through the reciprocal space (black bars), intensity traces of the cc peak (b) were extracted

the $q_{z}$ component. Depending on the angle of beam incidence the sample horizon was partially shadowing the intensity profiles at $\phi<8^{\circ}$ for some of the samples. Therefore, the Lorentzian fits for $\phi=8^{\circ}$ (Fig. 5a) were used for the calculations of $a$ and $\xi$ (Fig. 5b). In addition to the acyl chain distance $a$ and the correlation length $\xi$, the extension of the cc peak in $\Delta \phi$ gives information about the tilt homogeneity of the acyl chains (Fig. 4a, inset). Quantitative fits of the profiles along this dimension, as performed for pure lipids (Weinhausen 2010), were not applicable for the present data for reasons of the signal-tonoise ratio.

\section{$R H$ dependency: evaluation of the experiment}

First, it is important to keep in mind that the next neighbor distance $a$ between lipid acyl chains as well as the 
Fig. 5 The intensity profiles (Fig. 4b) at a certain constant $\phi$ were fitted to Lorentzian lineshapes (Eq. 2). The resulting fits at $\phi=8^{\circ}$ (a) were applied to calculate the mean lipid acyl chain distance $a$ and the acyl chain correlation length $\xi$ (b)

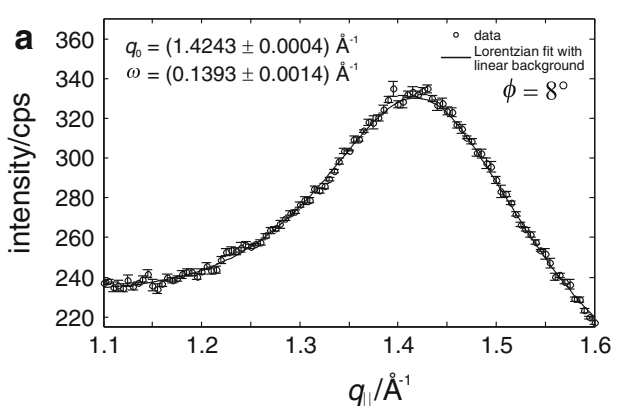

associated lipid chain correlation length $\xi$ are mean values averaged over the entire lipid bilayer stack illuminated by the X-ray beam. The acyl chain distance $a$ reflects the lateral lipid packing. Smaller values for $a$ correspond to a denser packing. The lipid ordering (short range order) is expressed by the acyl chain correlation length $\xi$. The obtained values for $a$ and $\xi$ are presented in Table 1 and visualized in Fig. 5b.

As we were interested in the changes of the lipid bilayer structure upon peptide insertion we had to exclude influences on the ordering and packing of lipids exerted by other parameters. These are, on the one hand, the constitution of the lipid species in terms of chain length and saturation (Seelig and Seelig 1977), chain branching (Perly et al. 1985), and headgroup structure (Cullis et al. 1986), and on the other hand, the lipid phase state (Lafleur et al. 1990b; Perly et al. 1985; Sternin et al. 1988) and the cholesterol content (Dufourc et al. 1980; Oldfield et al. 1978; Stockton and Smith 1976). The use of only one lipid component with a fixed cholesterol content eliminates the mentioned impacts. The uniformity of physical parameters, namely the temperature (Davis et al. 1980) and the relative humidity, was assured by the controlled sample environment.

Therefore, the experiments carried out with the isomorphic sample 3/6 (equimolar)/DLPC/Chol (1/38/2) at different values for $\mathrm{RH}$ (samples 5-9) served as a control for the suitability of the experimental protocol as well as the scattering setup and the data treatment. Furthermore, it should be tested whether a variation of $\mathrm{RH}$ is detectable by changes in $a$ and $\xi$ and if these changes are within an appropriate regime and follow expected trends.

In general, lowering the $\mathrm{RH}$ promotes the gel phase character of the lipid membranes causing a reduced fluidity and increased inter-bilayer potentials (Ho et al. 1995; Long and Hruska 1970). Upon reducing the water content of the system, the fraction of gauche bonds within the lipid acyl chains is diminished and leads to lipid chain stretching. As a consequence, the lipid chain alignment and ordering (Cevc and Marsh 1985) as well as a lateral compression or packing (Bryant et al. 2001; Chen and Hung 1996) are enhanced. Such behavior was confirmed by the results obtained from measurements for samples 5-9. In line with the literature, a lowering of the acyl chain distance of about $\Delta a_{\max }=0.097 \pm 0.006 \AA$ and an increase in the correlation length of $\Delta \xi_{\max }=0.8 \pm 0.2 \AA$ were observed when comparing the results for $\mathrm{RH}=94 \%$ to $\mathrm{RH}=25 \%$ and to $\mathrm{RH}=60 \%$, respectively. The revealed effect that at low water content with RHs between 40 and $25 \%$, the correlation length $\xi$ seems to reach saturation and even slightly decreases for sample 9, can be associated with the steric demands exerted by the peptide and cholesterol inclusions (Fig. 5b). Steric demands of the relatively rigid peptide helices become more critical in the more condensed phases than in the fluid phase (Marčelja 1974). This condition of so-called intermediate fluidity (Oldfield and Chapman 1972) describes the fact that the interaction between inclusions such as cholesterol or peptides cannot be as strong as interactions among ordered all-trans lipid chains (Marčelja 1974). We did not observe the phenomenon of an interchain hydration at high RH that is concomitant with an increased lipid chain-chain distance and results from defect structures caused by inserted peptides (Ho et al. 1995).

\section{The parameters $a$ and $\xi$ upon peptide insertion}

As a general trend upon reconstitution of the different peptide species, we observed a significant decrease in the acyl chain distance $a$ while the values for the lipid chain correlation length $\xi$ increased (Fig. 5b). One may initially expect the opposite effect, i.e., an increase in $a$ and a decrease in $\xi$. This could appear conclusive, since the occupation of the lipid bilayer area by peptide inclusions would lead to additional and longer trans-helix chain-chain distances for so-called annular lipids, which are in direct contact with the peptide helix. Furthermore, the absent chain-chain correlation between those lipids could reduce $\xi$. From the experimental results it is obvious that this is not the case, or at least other phenomena are outweighing such assumed effects leading to an increase in lipid packing (density) and chain ordering.

Changes in the lateral bilayer structure upon peptide insertion are mostly discussed in theoretical studies and appear contradictory with respect to their conclusions. 


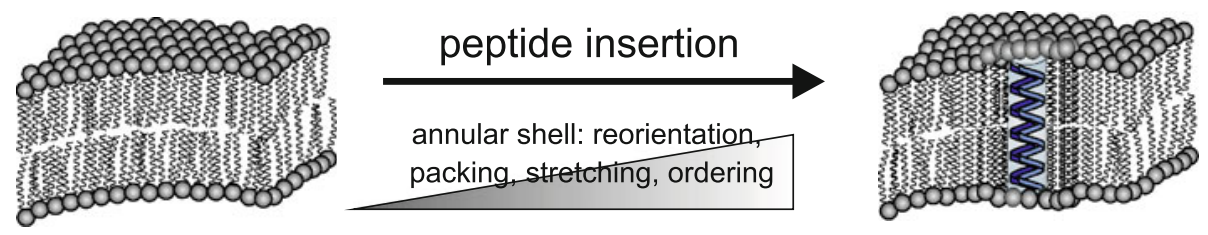

Fig. 6 Schematic representation of the conclusions drawn from GID results, suggesting a positive hydrophobic mismatch and, most likely, an enhanced highly ordered packing of the annular lipid shell. These

Already in early work, motivated by fluorescence data, a distinction is made between lipids not in contact with protein or peptide surface and lipids 'coupled' to the reconstituted protein, which are, thereby, disturbed in their interaction (Träuble and Overath 1973). These so-called annular lipids were shown not to undergo lipid phase transitions. The postulated protein-derived disturbance was calculated to extend up to the third lipid neighbor of a protein inclusion (Marčelja 1976). Addressing the structure of annular lipids in greater detail by theoretical molecular field approximations revealed an increased ordering of the lipid chains in proximity to membrane-incorporated structures within the fluid lipid phase (Marčelja 1974). With increasing fraction of "foreign molecules" on the phospholipid bilayer, the membrane order parameter was reported to increase and the dependency of the order parameter on temperature to become less pronounced. We will denote this scenario as the (annular) lipid ordering scenario. In the lipid condensed phase, this effect was described to be the opposite, which we denote as the lipid disordering scenario. Other authors claim that rigid inclusions such as cholesterol might cause straightening and ordering of lipid chains, while the fluid-like surface of embedded proteins does not influence the motional freedom of the lipid chains (Lafleur et al. 1990a). Molecular dynamics simulations of three different peptide $\alpha$ helices, published at the same time, likewise revealed an ordering of the lipid chains close to the peptide helices for all peptide species (Edholm and Johansson 1987). Even if the overall effect was assessed as "not drastic," the annular lipid ordering could be estimated as "much less pronounced" for lipids with bulky side chains. More recent theoretical considerations applying statistical mechanical integral equation theories (Lagüe et al. 1998) predict an expansion of the area per lipid for the annular shell upon peptide insertion into different phosphatidylcholine (PC) bilayers. In these studies, peptides are treated as soft cylinders (Lagüe et al. 2001). The authors postulate effective long range repulsion between lipids and peptides due to the formation of a lipid depletion layer around a protein leading to an increased cross-sectional area per lipid molecule. According to this view, which also supports the lipid effects may be attributed to the specific properties of the peptide species, i.e., their sequences, side-chain orientations, and secondary structures

disordering scenario for annular lipids, a lipid molecule in close contact with the peptide must significantly reduce its order. Effective lipid-protein repulsion would arise due to its entropic disadvantage. These theoretical results were further supported by MD simulations of a hydrated 1,2-diphytanoyl-sn-glycero-3-phosphocholine (DPhyPC) bilayer containing an $\alpha$-helical peptide bundle of four transmembrane domains (Husslein et al. 1998). Comparable to the results of Laguie et al., Husslein and coworkers revealed an increase in the area per lipid from $7.46 \mathrm{~nm}^{2}$ for the unperturbed bilayer to $8.50 \mathrm{~nm}^{2}$ in the vicinity of the peptide species. Finally, for the sake of completeness, we note that a pronounced annular lipid effect is not reported by all studies. Using spin labeling techniques, Marsh and Horváth found order parameters of protein-associated lipid chains to be very similar to those in the bulk liquid-crystalline phase regions (Marsh and Horváth 1998).

Considering these opposing scenarios, it is interesting that we observed a very clear indication of the lipid ordering scenario. The decrease in the average lipid chain distance $a$ as well as an increase in the lipid chain correlation length $\xi$ for the peptides studied here can be ascribed to a reduced number of lipid gauche rotamers (Fig. 6). For different molecular systems imposing different molecular boundary conditions, the second scenario could be valid instead. It should be taken into consideration that for the sake of simplicity, the theories supporting either one of the scenarios neglect the individual chemical properties of the incorporated molecular species that are the bases for interaction at the peptide/lipid interface.

However, the particular molecular properties of different species may be the decisive parameters that determine whether a peptide/lipid interaction is attractive or repulsive. For the presented peptide species 1-6 these parameters are (1) the unique $\beta$-helical secondary structure caused by the D,L-alternation and bulkiness of the peptide side chains, (2) the exclusiveness of the side-chain composition with almost entirely aromatic amino acid residues, and (3) the presence of flanking interfacial anchoring moieties. Our findings are also in line with literature studies that report higher order parameters for hydrocarbon chains of lipids adjacent to peptide channels composed of aromatic amino 
acids (Chiu et al. 1999). This should be particularly pronounced in the case of tryptophan-containing peptides, such as species 1-9, as tryptophan is known as an amino acid with high preference for lateral interaction (Adamian and Liang 2001; Ridder et al. 2005).

The planarity and hydrophobicity/amphipathicity of the aromatic side chains have already been shown to provide potential for a perfect alignment and interaction with the lipid bilayer core via intercalation between the lipid chains (Hite et al. 2008; Palsdottir and Hunte 2004). Hydrophobic interaction might further direct lipid chains to approach closer contact to incorporated peptide species via lowering entropic costs for acyl chain deformation. This lipid/peptide interaction might play a predominant role for $\beta$-helical peptide species due to the fact that all peptide side chains are pinpointing radiantly outside the helix (Koert et al. 2004). Proper vertical alignment for the reconstituted peptide in the bilayer was concluded already in a previous reflectivity study exhibiting up to six lamellar orders nearly independent of the applied peptide concentration (Küsel et al. 2007).

Owing to the nature of bilayer elasticity, the effects of lateral packing cannot be discussed without addressing the vertical structure and density profile, which are closely interrelated. Namely, the effects of hydrophobic matching and interfacial anchoring (Killian and Nyholm 2006) interfere with the lateral forces. Hydrophobic matching and interfacial anchoring are especially important for the presented peptide species, as all of them hold two flanking tryptophan residues. The magnitude of hydrophobic mismatch is considered to be a function of the distance between interfacial anchoring residues, here tryptophans, and not the overall peptide length (De Planque and Killian 2003). In this respect, tryptophan residues are known to provide the highest tendency for localization at the interface between the lipid bilayer core and the polar lipid headgroup. Hydrogen bonding and electrostatic interactions are discussed to account for the interfacial anchoring and $z$ positioning of membrane-incorporated peptides (De Planque and Killian 2003; Doux and Killian 2010).

A stretching of the lipid bilayer in the $z$ direction caused by hydrophobic mismatch (Killian and Nyholm 2006) may alone result in pronounced lateral effects on annular lipids. Huang and coworkers observed a stretching of a DLPC lipid bilayer upon incorporation of gramicidin A that likewise adopts $\beta$-helical structures, similar to the $\mathrm{D}, \mathrm{L}$-alternating peptides applied in the presented approach (Harroun et al. 1999). In previous studies of the homodimeric species $\mathbf{1}$, comparable effects were indicated by a thickening of the lipid bilayers of $1.4 \AA$ (DMPC $=1,2$-dimyristoyl-sn-glycero-3-phosphocholine) and $4.0 \AA$ (DLPC), respectively (Küsel et al. 2007). Finally, the reflectivity experiments of the designed peptide hairpin structures $2,5,7,8$, and 9
Table 2 Lipid bilayer thickness upon peptide insertion. Repeat spacing and peak-to-peak distances (electron density profiles) of the pure lipid samples and different peptide/lipid complexes as revealed by in-house reflectivity studies suggesting a positive mismatch situation

\begin{tabular}{lll}
\hline Sample composition & $\begin{array}{l}\text { Repeat spacing } \\
d(\AA)\end{array}$ & $\begin{array}{l}\text { Peak-to-peak } \\
\text { distance } \sim d_{\mathrm{PP}}(\AA)\end{array}$ \\
\hline DLPC/Chol (95/5) (pure lipid) & $45.45 \pm 0.34$ & 30.8 \\
DLPC/Chol/2 (47.5/2.5/1) & $46.25 \pm 0.39$ & 31.4 \\
DLPC/Chol/5 (47.5/2.5/1) & $46.73 \pm 0.20$ & 32.4 \\
DLPC/Chol/7 (47.5/2.5/1) & $46.50 \pm 0.05$ & 31.6 \\
DLPC/Chol/8 (47.5/2.5/1) & $46.71 \pm 0.15$ & 32.2 \\
DLPC/Chol/9 (47.5/2.5/1) & $46.89 \pm 0.09$ & 31.8 \\
\hline
\end{tabular}

All data were obtained at relative humidity of $94 \%$

unambiguously prove a positive mismatch situation, also for the hairpin structures, as presented in Table 2. Such mismatching promotes stretching of the lipid chains and reduction in gauche conformations in the lipid acyl chains. The mismatch situation as well as the described lateral effects may be directly coupled or, alternatively, may independently contribute to the observed changes in $a$ and $\xi$ upon peptide insertion, as addressed in the following section.

\section{Differences in peptide species: the cross-sectional area and the characteristics of peptide/lipid contacts}

If the ordering of the lipid chains is solely a function of hydrophobic mismatch, any of the studied peptide species should yield the same values for $a$ and $\xi$ due to sequence similarity. Obviously, this is not the case (Fig. 5b).

For the sample solely composed of DLPC and cholesterol (sample 1) the acyl chain distance is $a=4.94 \pm 0.01 \AA$. The shortening of the acyl chain distance $a$ appears more pronounced for the homodimeric species $\mathbf{4}$ (sample 2) at a helix-to-lipid ratio of $1 / 20(\mathrm{P} / \mathrm{L}=1 / 10)$ but is even stronger for the hairpin species 5 at the same helix-to-lipid ratio $(\mathrm{P} / \mathrm{L}=1 / 20)$. Considering the recognition system $\mathbf{3 / 6}$ (equimolar) at $\mathrm{RH}=94 \%$ (sample 5), the shortening of $a$ is less distinct but can also be assigned to a lower helix-to-lipid ratio of $1 / 40(\mathrm{P} / \mathrm{L}=1 / 20)$. The concentration effect can be factored out by comparing different samples ( 3 and 4 ) of the hairpin species at $\mathrm{P} / \mathrm{L}=1 / 20$ and $\mathrm{P} / \mathrm{L}=1 / 40$, respectively, showing that higher peptide concentration yields shorter lipid chain distances. It can further be concluded that the recognition system 3/6 (equimolar ratio) at $\mathrm{RH}=94 \%$ (sample 5) has less impact on $a$ than the respective hairpin peptide 5 (sample 4 ) at identical $P / L=1 / 40$. The values for the lipid chain correlation length $\xi$ follow an almost opposing trend as that observed for $a$. Peptide incorporation generally leads to an increase in $\xi$. 
In principle, the homodimer (4), hairpin (5), and recognition system (3/6) structures are designed to adopt an identical secondary structure with respect to their TMDs. In the case of the hairpin structure 2 , due to a decline in helix propensity compared to the homodimeric species $\mathbf{1}$ as revealed by CD spectroscopy (Schneggenburger et al. 2010), it was assumed that the hairpin structure exhibits steric clashes in the reverse-turn region leading to a less perfect alignment of the double strands and a broadening of the helix diameter. Therefore, the self-associated homodimer 4 should provide a closer alignment enabled by hydrogen bonding of the two antiparallel-oriented strands. The PNA recognition system $\mathbf{3 / 6}$ leading to an aggregation of two of the double helices in a close contact state (Schneggenburger et al. 2010) would yield more than a doubled lateral occupied area within the lipid bilayer (Fig. 2c). Under these circumstances, the lateral dimensions of the peptide species are supposed to increase in the order: homodimer (4) $\rightarrow$ hairpin (5) $\rightarrow$ recognition system $(\mathbf{3 / 6})$.

As the differences in the values for $a$ and $\xi$ when comparing the different peptide species cannot be solely attributed to a hydrophobic mismatch situation (see above), lateral peptide parameters such as the peptide cross-sectional area or the peptide/lipid contact surface are assumed to be the primary cause for the observed differences. In line with the lipid disordering scenario discussed above, larger peptide cross-sectional areas and peptide lipid contact surfaces would probably cause higher values for the lipid acyl chain distance $a$ and smaller values for the lipid correlation length $\xi$. This is true if peptide/lipid interactions are neglected or a repulsive potential between lipids and peptides is assumed (Lagüe et al. 2001). For the present sample sequence: homodimer (4) $\rightarrow$ hairpin (5) $\rightarrow$ recognition system (3/6), this would imply an increase in $a$ and a decrease in $\xi$. The fact that the observed results point to the opposite may be taken as evidence for attractive peptide lipid interactions. Due to the sequence identity of the antiparallel TMD strands of all peptide species, and therefore, the constitutional similarity of the postulated attractive peptide/lipid interactions, only the peptide secondary structure, i.e., the lateral sizes of the peptides, and the size of the lipid/peptide interfacial plane should affect $a$ and $\xi$. Therefore, a monotonous decrease in the lipid chain distance would be expected for samples $1-3$, which is in line with the obtained results (Fig. 5b). On the contrary, a monotonous increase in the chain correlation length was not observed. The value for $\xi$ of the homodimeric species 4 (sample 2) appears higher than that for the hairpin $\mathbf{5}$ (sample 3). This might be explained by the fact that oligomer 4 is known to experience a monomer-dimer equilibrium (Küsel et al. 2007) including a structural change from a membrane-spanning $\beta^{5.6}$ helix to two reasonable broader and shorter $\beta^{6.3}$ single helices (Fig. 2). This would actually change the peptide dimensions in the $z$ direction as well as the molecular constitution of the helix surface and therefore cause different hydrophobic mismatch situations as well as changed lateral peptide/lipid interactions.

In the case of the recognition system $\mathbf{3 / 6}$, a close contact state along the outer helix shells results from PNA pairing and TMD assembly (Schneggenburger et al. 2010). The adopted peptide complexes can be considered as one reconstituted peptide species surrounded by annular lipids (Fig. 2c). The recognition process, therefore, diminishes the peptide/lipid contact area by shielding the inner helix flanks towards the lipid matrix. In this respect, the recognition system species $\mathbf{3 / 6}$ (sample 5) provides lower values for $a$ and higher values for $\xi$ than the hairpin species $\mathbf{5}$ at the same $\mathrm{P} / \mathrm{L}$ ratio.

Peptide positioning and orientation from reflectivity using iodine labels

The membrane response in terms of changes in the lipid correlation length and lipid chain distance addressed above can neither reveal the insertion depth of a particular peptide side chain nor does it allow distinguishing between a membrane-spanning state or insertion perpendicular to the membrane normal. FRET experiments (parallax analysis) are well suited to studying membrane proteins in vivo but are often limited in resolution caused by large Förster radii of the fluorescence probes. In contrast, X-ray reflectivity in combination with contrast variation by heavy atom labeling can provide structural constraints down to subnanometer resolution for fluid or condensed peptide/lipid samples. For a fast in-house approach, the analysis of the electron density difference is a unique method to obtain highly resolved information about the localization of a certain structural element within a lipid bilayer (Fig. 7). As shown in previous studies for the double helical homodimer 1, this method is even capable of producing evidence of a transmembrane peptide orientation within the lipid bilayer via iodine labeling at several positions (Küsel et al. 2007). With the iodine-labeling approach, the alignment of the membrane-spanning helical hairpin formed by the severe acute respiratory syndrome (SARS) coronavirus E protein could be elucidated by localizing the Phe 23 adjacent to the lipid headgroup region (Arbely et al. 2004; Khattari et al. 2006a, b). Instead of determining the label position by a comparison of the deduced electron densities $\rho(z)$ resulting from unlabeled and labeled samples, this can also be accomplished using an isomorphic sample and applying different photon energies (Khattari et al. 2005).

In this work, we used both approaches, the label replacement by a sample series for in-house reflectivity and the contrast variation via anomalous reflectivity for the synchrotron studies. In both cases, the commercially 
Fig. 7 Schematic representation of the reflectivity scattering geometry for multilamellar P/L complexes (a) with highlighted iodine positions (red arrows) and deduced electron density profiles (right, b). The comparison of electron density profiles originating from samples containing labeled and unlabeled peptide species or from scattering at different photon energies yields the difference curve (blue)

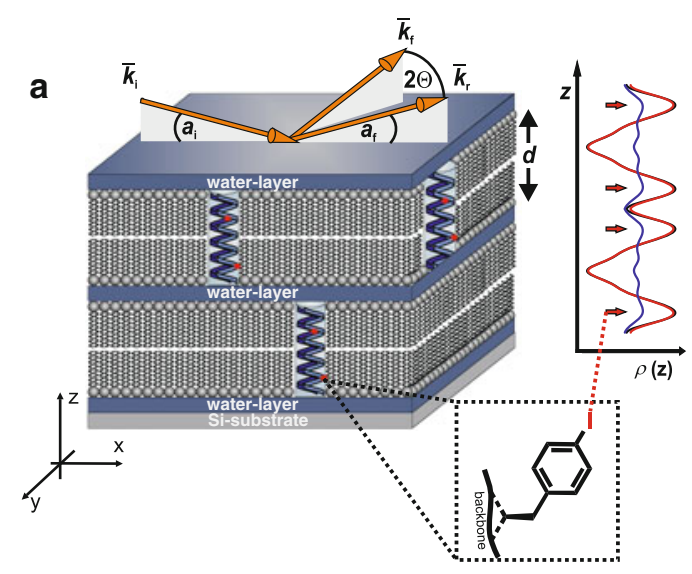

available building block Fmoc-Phe(4-I)-OH served as iodine label, substituting the native phenylalanine.

Within the centrosymmetric electron density profiles deduced from reflectivity scans (Fig. 7b), maxima correspond to the lipid headgroup regions, while the global minimum reflects the acyl-acyl contacts of opposing hydrocarbon chains. The adjacent water layers are represented by the side minima. For anomalous scattering, the electron density profiles obtained at the $L_{\mathrm{III}}$ absorption edge $(E=4.5578 \mathrm{keV})$ were subtracted from the electron density profiles at $E=5.8000 \mathrm{keV}$ to estimate the position of the iodine label with respect to the $z$ direction, indicated by rises in the electron density difference curve (Fig. 7b, blue curve). In in-house experiments such curves result from subtraction of the electron density $\rho(z)$ of the samples containing iodinated peptides from the sample with unlabeled peptide species.

The detection of single iodine labels via difference analysis usually requires high peptide-to-lipid ratios in the range of $\mathrm{P} / \mathrm{L} \approx 1 / 10$ (Arbely et al. 2004; Küsel et al. 2007). With the synthesis of the novel double iodinated amino acid building block Fmoc-5,5-diiodoalyllglycine$\mathrm{OH}$, the use of an enhanced in-house reflectivity set up including substrate sizes of $15 \times 25 \mathrm{~mm}$ and the addition of $5 \mathrm{~mol} \%$ cholesterol to the lipid phase enabled recording up to seven lamellar orders from samples with a $\mathrm{P} / \mathrm{L}=$ $1 / 50$. The analysis of the electron density difference profiles then allowed for the determination of the iodine positions with high accuracy (Schneggenburger et al. 2009). Even for the Fmoc-Phe(4-I)-OH single label, P/L ratios of 1/50 turned out to be sufficient. Therefore, in-house reflectivity studies and anomalous reflectivity using synchrotron radiation were both performed at $\mathrm{P} / \mathrm{L}=1 / 50$. Three differentially labeled peptide species, 5, 7, and $\mathbf{8}$ (Fig. 8), were used to test for a transmembrane orientation. To avoid beam damage during anomalous reflectivity experiments, the respective curves were solely scanned around the Bragg reflections.

Four to five lamellar orders (Fig. 9a) were recorded by specular and offset (longitudinal diffuse) scans. Unfortunately, the specular condition (angular alignment) was lost in some of the scans, so that the diffuse offset scans, which by nature are less sensitive to angular misalignment and which show strong lamellar reflections, were used for the Fourier synthesis analysis. Due to the difficulties associated with low energies around the I- $L_{\mathrm{III}}$ edge and the intrinsically small anomalous difference signal, all experiments were repeated on an in-house diffractometer without any risk of radiation damage during long scanning and with most careful alignment procedures. The one-dimensional swelling method was used to yield the phases $v_{n}$.

In in-house experiments, six intensive equidistant Bragg peaks were obtained for the labeled (7) as well as the unlabeled (2) hairpin species (Fig. 9b) due to the higher radiation energies $\left(\mathrm{Cu}-K_{\alpha}=8.048 \mathrm{keV}\right)$ and improved alignment. For peptides labeled with the Fmoc-Phe(4-I)$\mathrm{OH}$ building block, species $\mathbf{2}$ served as reference structure. In case of hairpin 8, labeled with Fmoc-5,5-diiodoallylglycine- $\mathrm{OH}$, analog 9 with the sequence $\mathrm{H}-(\underline{\mathrm{FY}})_{5} \underline{\mathrm{WW}}-\mathrm{G}-$ allylglycine-PG-(FY) ${ }_{5}$ WW-OH was applied as unlabeled species. As the electron density profiles of the lipid bilayer structures obtained from in-house experiments were more resolved, these data were used to estimate the respective changes in hydrophobic thickness (see below).

The X-ray reflectivity curves (Fig. 9) and the corresponding electron density profiles (Fig. 10a, b) are exemplarily shown for compound $\mathbf{7}$ and for its unlabeled analog 2 (in-house), respectively. More data are shown in the Electronic Supplementary Material. The derived electron density difference curves for all studied constructs $\mathbf{5}$, $\mathbf{7}$, and $\mathbf{8}$ and unlabeled compounds $\mathbf{2}$ and $\mathbf{9}$ are depicted in Fig. 10b, c. 
Fig. 8 Chemical structures of iodine-labeled hairpins $\mathbf{5}, \mathbf{7}$, and $\mathbf{8}$, as well as their schematic sketches (right)
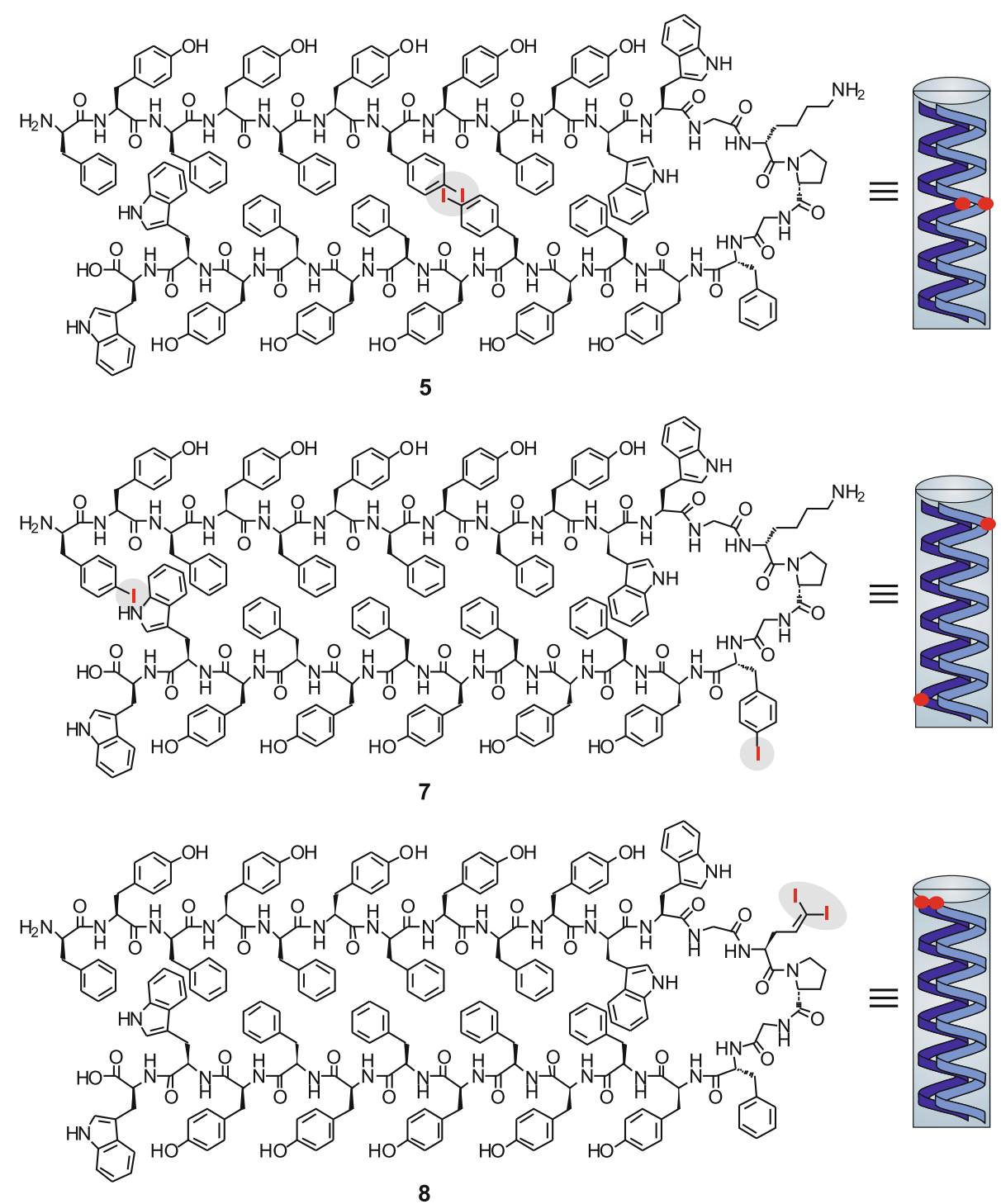

8

Fig. 9 Illustrative data curves for diffuse anomalous reflectivity (a) and in-house reflectivity (b), shown for the 7/DLPC/Chol (1/47.5/2.5) sample. For the synchrotron data recorded at low energies (iodine $L_{\mathrm{III}}$ edge), the scattering curves were solely recorded around the Bragg reflections, since sample degradation by beam damage was observed after extended scanning

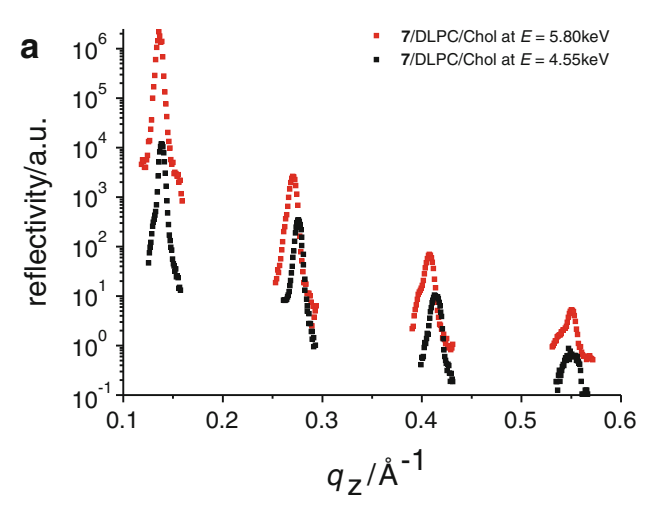

\section{Membrane thickening and hydrophobic mismatch}

The reflectivity curves and deduced electron density profiles reveal a stretching of the lipid bilayers upon peptide insertion. This was found for all examined peptide species $(\mathbf{2}, \mathbf{5}, \mathbf{7}, \mathbf{8}, \mathbf{9})$ and is in line with the obtained GID results confirming a positive hydrophobic mismatch. The repeat spacing and the peak-to-peak distance $\left(d_{\mathrm{pp}}\right.$, compare to Fig. 7) values (Table 2) evidence a small thickening of the lipid bilayer up to $1.4 \AA$, which is more pronounced for the 
Fig. 10 Derived electron density curves for 7/DLPC/Chol (1/47.5/2.5) from diffuse anomalous scattering (a) and inhouse reflectivity $(\mathbf{c})$. In the inhouse experiments the samples containing the iodine-labeled compound 7 were compared to the samples lacking the iodine probes (compound 2, black curve). The electron density difference curves (blue, shifted for clarity) are depicted for all peptide constructs 5,7 , and 8 either studied by synchrotron radiation (b) or by in-house reflectivity (d). The curves derived from in-house measurements are multiplied by a factor of 3 for clarity; the headgroup region is highlighted (green area)
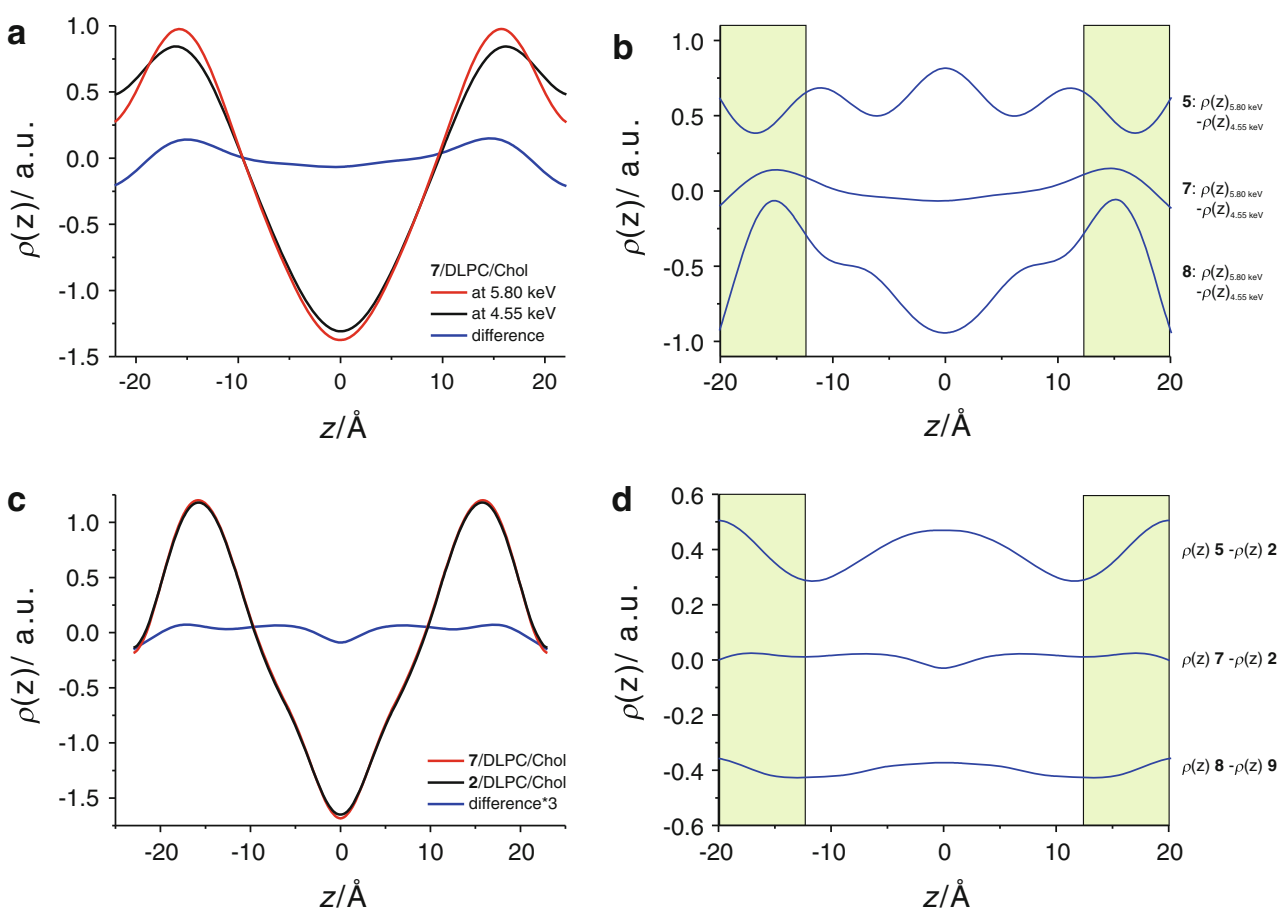

mono-iodinated species 5 and 7. In case of the doublelabeled species $\mathbf{8}$ and its reference analog $\mathbf{9}$, this effect is inverted.

In general, the electron contrast variation resulted in changes in the headgroup densities, which were too pronounced to be explained by the contrast variation itself and therefore indicate systematic errors. These probably stem from misalignment or drift when changing the photon energies. Thus, the two approaches of contrast variation (in-house and synchrotron) are not in quantitative agreement. For the in-house measurement, the effect of a decrease in electron density contrast of the side minima compared to the global minimum is observed in most peptide/lipid systems. The decrease can be explained by a beginning lipid disorder that evens the density profile by increased density in the water layer and/or an enlarged area per headgroup (Li and Salditt 2006); this effect may be more pronounced for a peptide carrying a bulky iodine label. However, we also cannot rule out systematic errors for the in-house measurements, and beyond the thickening effect, which appears to be robust, the small differences between curves with and without labels may impede an unambiguous localization of the iodine position. Finally, we must be cautious since iodine-labeling itself may influence peptide/lipid interactions. However, there is no obvious reason why attachment of the iodine labels at different positions should cause different peptide positioning, especially as the iodine labels have been shown to have only minor effects on the peptide secondary structure (Schneggenburger et al. 2009).
Notwithstanding the problems mentioned above, we will now address the difference curves in view of a tentative interpretation of label position and peptide orientation in the next subsection, step by step for each peptide and labeled construct.

\section{Hydrophobic mismatch and peptide orientation of single species}

Species 5 The center maximum of the density difference curve for the middle-labeled oligomer 5 (both intertwined strands) indicates that a significant fraction of the iodine labels are buried deeply in the hydrophobic core of the lipid bilayer (Fig. 10b, d). In both data sets, in-house and synchrotron, the membrane thickness (peak-to-peak distance) is approximately $32 \AA$ (see the Electronic Supplementary Material). The distribution of the central maximum of the difference curve appears relatively broad, especially for the in-house experiment (Fig. 10c).

The synchrotron data do not only illustrate a rise in intensity at the bilayer center but also at approximately $z= \pm 11.1 \AA$, which does not occur in the higher resolution in-house study (Fig. 10b, d). These peak positions may still be commensurate with more than one orientation, for example either a membrane-spanning orientation of the $\beta$-hairpin (Fig. 11i) or an orientation of the peptide species parallel to the membrane surface (Fig. 11ii) at the bilayer center. The latter is, however, not very likely for energetic reasons. 
Species 7 Anomalous reflectivity of terminally labeled peptide species 7 in peptide/lipid complexes (Fig. 10a) showed relatively weak minima in the density corresponding to the water layer. If this is not an artifact of the rather problematic synchrotron experiment, it could be possibly attributed to increased lipid fluctuations or beginning lipid disorder upon localization of the rather bulky iodine labels at the lipid headgroup region. The electron density difference curve shows only two symmetrically distributed maxima at approximately $z= \pm 15.0 \AA$ indicating a localization of the iodine labels at the lipid headgroup region tending to the membrane interior. This observation would perfectly fit with a transmembrane orientation of the $\beta$-hairpin (Fig. 11i), although an orientation parallel to the membrane surface at the headgroup region (Fig. 11iii) cannot be ruled out. Taking into account the observations made for the peptide/lipid complex 7/DLPC/Chol (1/47.5/ 2.5), this appears rather unlikely, since the depth of the insertion should not depend on the position of the label along the helix. The distance between the maxima of the electron density difference curve, associated with the distance of the two iodine labels is about $32 \AA$ (see the Electronic Supplementary Material). This is in good agreement with the expectation based on a peptide length of $26 \AA$ and the additional spacing of the Phe(4-I) side chains attached at both ends of the double helix and pointing to opposite directions (Schneggenburger et al. 2009).

The electron density profiles derived from the in-house measurements of compounds 7 and 2 (Fig. 10c) show reasonable lower contrast between labeled and unlabeled samples, which is much closer to the expectation. Four small bumps in the electron density difference curve appear at $z= \pm 17.0$ and $\pm 7.0 \AA$ (Fig. 10d), corresponding to possible inter-iodine distances of approximately $\Delta z=34$, 14,10 , or $24 \AA$. Compared to $\beta$-structures of gramicidin $\mathrm{A}$

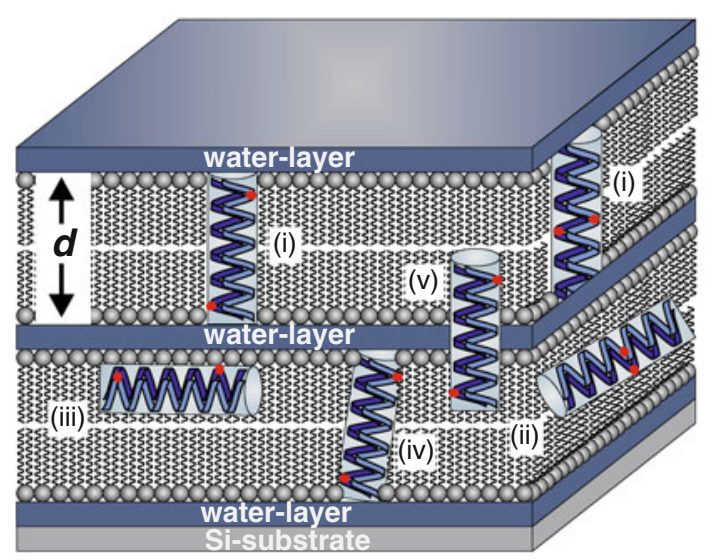

Fig. 11 Exemplary schematic representation of orientations that could be adopted by labeled peptide species in lipid multilayers and fit the reflectivity data of one or the other peptide species that are likewise assumed for peptide 7 or the unlabeled analog 2 with a periodicity of 5.6 or 6.3 , the investigated $\beta$-hairpin with a strand length of 12 residues would give a peptide length of 28.8 or $20.8 \AA$, respectively (Fahsel et al. 2002). This would not directly match a transmembrane orientation without assuming additional effects from distortions, tilt (Fig. 11iv), or a coexisting population (Fig. 11v). The rather flat difference curve of the in-house data for species 7 appears less conclusive than difference curves with more clearly identifiable maxima.

Species 8 In case of the molecular species $\mathbf{8}$, holding a 5,5-diiodo-allyglycine label at the loop position, five lamellar orders could be obtained in the synchrotron experiment (see the Electronic Supplementary Material). The peak-to-peak distance of the electron density curves reveals a headgroup spacing of approximately $33 \AA$ (4.55 keV) and $32 \AA(5.80 \mathrm{keV})$, respectively. The corresponding electron density difference curve is pronounced due to the double signal intensity resulting from the double iodinated label, but differences in resolution at the different $\mathrm{X}$-ray energies may also play a role. The profile shows strong maxima at $\pm 15.4 \AA$ and additional inflection points around $\pm 7.1 \AA$ (Fig. 10d). The maxima at $\pm 15.4 \AA$ may indicate a transmembrane orientation of the $\beta$-hairpin structure $\mathbf{8}$ nearly spanning the same distance as the terminally labeled sample 7/DLPC/Chol. The electron density difference curve of in-house reference measurements does not show significant rises in the headgroup region, but only at the membranes outside $( \pm 21.4 \AA)$ in the membrane water layer (see above). A rather diffuse and less intense maximum appears spread out over the bilayer hydrophobic core.

Summarizing the results of the iodine-labeling experiments, the electron density difference data from synchrotron experiments and in-house data are not in agreement. While the synchrotron experiment faced technical challenges related to the relative low photon energy ( $L_{\mathrm{III}}$ edge) and sample realignment after beam drift when changing the energy, as well as intrinsically lower contrast, the comparison of two different, not necessarily isomorphic, samples in the in-house study may also pose a challenge. In other words, the differences from one sample to the next may be on the same order as the label effect itself. Note that one needs a much higher reproducibility to interpret the difference curve than the rather gross features of the profiles before subtraction. Therefore, we consider the results regarding the membrane repeat spacing to be more reliable and consistent than conclusions drawn from the peptide labels.

Fortunately, however, the reflectivity results for the different peptide species 5, 7, and $\mathbf{8}$ need not be judged alone to make conclusions about the peptide orientation 
(Fig. 11). The observed changes of the inner hydrophobic core upon peptide insertion, as revealed by GID/reciprocal space mapping, together with the sum of the reflectivity results may not completely rule out a simple surface anchoring or surface adhesion of the peptide species 2-9, but they make an inserted orientation parallel to the membrane normal, possibly with some tilt distribution, much more likely. This would be further supported by band shifts of the fluorescence emission spectra (Schneggenburger et al. 2010), indicating a localization of flanking tryptophan residues in the lipid headgroup regions.

\section{Concluding remarks}

The lipid response upon the insertion of peptide constructs systematically varied in the oligomerization state and secondary structure was addressed by an X-ray scattering study. The approach included grazing incidence diffraction and X-ray reflectivity and is, in principle, applicable to any multilamellar peptide/lipid complex.

The preparation of multilamellar lipid samples is pretty much straightforward and only requires minor adjustments due to solvent compositions at high $\mathrm{P} / \mathrm{L}$ ratios. In contrast, the requirements for the sample environment are rather high in terms of a stable and reproducible RH. Nevertheless, an advanced setup is not mandatory, since very distinct and stable RHs can also be generated by applying saturated salt solution reservoirs in a sealed sample chamber. (Greenspan 1977; Winston and Bates 1960).

It has been shown that reflectivity experiments can be carried out with an in-house diffractometer with high performance and advanced resolution. This is especially true for an analysis of the hydrophobic membrane thickness variation upon peptide insertion. For a labeling approach, presented herein, in-house reflectivity holds the advantage of a higher contrast variation compared to energy variation around the I- $L_{\text {III }}$ absorption edge but includes the application of nonisomorphic samples. However, in-house reflectivity in combination with heavy-atom labeling is generally suited to generate reliable results for peptide side-chain positioning within the lipid bilayer (Küsel et al. 2007; Schneggenburger et al. 2010).

GID experiments are normally not only carried out at beamline experimental stations; a more extended sample screening appears only reasonable for taking advantage of the relatively short exposure times resulting from usage of a high-brilliant synchrotron X-ray beam. The methods presented herein are amenable to users that are not closely related to the X-ray field. This is notably true, since macro scanning and automated data evaluation can be applied that is even recommended if it is dealt with an increased quantity of samples.
In this study GID yielded quantitative values for the lipid chain correlation length $\xi$ and the average lipid chain spacing $a$ via analysis of the lipid chain correlation peak. Reflectivity studies provided additional information about the variation in the lipid spacing $d$ and bilayer thickness $d_{\mathrm{PP}}$ (Fig. 7b). This rather general approach of monitoring the collective lipid response to peptide interaction was combined with multiple site-directed iodine labeling, extending previous studies (Küsel et al. 2007; Schneggenburger et al. 2010) through a larger variety of constructs, labels, and label positions. While a good approach in principle, this part of the presented work showed results that were less consistent and thus less conclusive due to experimental difficulties. Future improvements could include the use of the $\mathrm{I}-\mathrm{K}_{\alpha}$ edge at $33.17 \mathrm{keV}$ rather than the low energy $L$ edges or the application of a more dedicated setup for this spectral range. Despite some of these problems, the data seem to point to a membranespanning orientation of the designed $\beta$-helical peptide hairpins.

Without relying on the contrast variation, a thickening of the bilayer by stretching of lipids in the $z$ direction could be clearly and consistently evidenced. Thus, membrane insertion of $\beta$-helical structures is characterized by a positive mismatch situation. This hydrophobic stretching of the membrane and a good accommodation of the peptide species, which might be based on their extraordinary sidechain composition, also result in an increased membrane packing and lipid ordering of annular lipids. These findings are in contrast to theoretical studies that describe peptide helices as soft cylinders (Lagüe et al. 1998, 2001). Two effects could possibly contribute to the enhanced lateral packing, the hydrophobic mismatch and/or hydrophobic, attractive lipid/peptide interactions resulting from sidechain intercalation into the lipid matrix.

Future experiments can be designed to evaluate the separate effects of lateral packing and ordering, systematically varying the mismatch by using longer chained lipids such as DMPC or reducing the aromatic content of peptide side chains via sequence mutation.

Acknowledgments We thank T. Reusch (Salditt group) for the development and programming of the humidity setup as well as writing the 'lamellar' software tool for data analysis. Further thanks go to P. Boeseke, D. Carbone, and T. H. Metzger for their great support during experiments at the ID01 beamline of ESRF, Grenoble, France. For completing the Göttingen beamtime crew, we thank S. Aeffner. We thank the scientific advisory board and the synchrotron laboratory for ample beamtime and for the opportunity to merge shifts of two projects. Generous financial support of the Deutsche Forschungsgemeinschaft (DFG) with the Collaborative Research Center (SFB) 803: 'Functionality controlled by organization in and between membranes' is gratefully acknowledged. P.E.S. is grateful for a bridging stipend of the Göttingen Graduate School of Neurosciences and Molecular Biosciences (GGNB). 
Open Access This article is distributed under the terms of the Creative Commons Attribution Noncommercial License which permits any noncommercial use, distribution, and reproduction in any medium, provided the original author(s) and source are credited.

\section{References}

Adamian L, Liang J (2001) Helix-helix packing and interfacial pairwise interaction of residues in membrane proteins. J Mol Biol 311:891-907

Aeffner S, Reusch T, Weinhausen B, Salditt T (2009) Membrane fusion intermediates and the effect of cholesterol: an in-house X-ray scattering study. Eur Phys J E Soft Matter Biol Phys 30:205-214

Alexopoulos E, Küsel A, Sheldrick GM, Diederichsen U, Usón I (2004) Solution and structure of an alternating D, L peptide. Acta Crystallogr Sect D Biol Crystallogr 60:1971-1980

Als-Nielsen J, McMorow D (2001) Elements of modern X-ray physics. Wiley, Weinheim

Andronesi OC, Pfeifer JR, Al-Momani L, Özdirekcan S, Rijkers DTS, Angerstein B, Luca S, Koert U, Killian JA, Baldus M (2004) Probing membrane protein orientation and structure using fast magic-angle-spinning solid-state NMR. J Biol NMR 30:253-265

Arbely A, Khattari Z, Brotons G, Akkawi M, Salditt T, Arkin IT (2004) A highly unusual palindromic transmembrane helical hairpin formed by SARS coronavirus E protein. J Mol Biol 341:769-779

Bechinger B (2001) Membrane insertion an orientation of polyalanine peptides: a ${ }^{15} \mathrm{~N}$ solid-state NMR spectroscopy investigation. Biophys J 81:2251-2256

Bong DT, Janshoff A, Steinem C, Ghadiri MR (2000) Membrane partitioning of the cleavage peptide in Flock House virus. Biophys J 78:839-845

Bryant G, Koster KL, Wolfe J (2001) Membrane behaviour in seeds and other systems at low water content: the various effects of solutes. Seed Sci Res 11:17-25

Bunkóczi G, Vértesy L, Sheldrick GM (2005) The antiviral antibiotic feglymycin: first direct-methods solution of a 1000+ equal-atom structure. Angew Chem Int Ed 44:1340-1342

Cevc G, Marsh D (1985) Hydration of noncharged lipid bilayer membranes. Theory and experiments with phosphatidylethanolamines. Biophys J 47:21-31

Chattopadhyay A, London E (1987) Parallax method for direct measurement of membrane penetration depth utilizing fluorescence quenching by spin-labeled phospholipids. Biochemistry 26:39-45

Chen FY, Hung WC (1996) Structural changes of lipid membrane induced by dehydration. Chin J Phys 34:1363-1372

Chen Z, Rand RP (1997) The influence of cholesterol on phospholipid membrane curvature and bending elasticity. Biophys J 73:267-276

Chen Y, Wallace BA (1997) The effects of calcium ions on double helical forms of gramicidin. Eur Biophys J 26:299-306

Chiu S-W, Subramaniam S, Jakobsson E (1999) Simulation study of a gramicidin/lipid bilayer system in excess water and lipid. I. Structure of the molecular complex. Biophys J 76:1929-1938

Chung LA, Lear JD, DeGrado WF (1992) Fluorescence studies of the secondary structure and orientation of a model ion channel peptide. Biochemistry 31:6608-6616

Constantin D, Mennicke U, Li C, Salditt T (2003) Solid-supported lipid multilayers: structure factor and fluctuations. Eur Phys J E Soft Matter Biol Phys 12:283-290
Constantin D, Brotons G, Jarre A, Li C, Salditt T (2007) Interaction of alamethicin pores in DMPC bilayers. Biophys J 92:3978-3987

Cullis PR, Hope MJ, Tilcock CPS (1986) Lipid polymorphism and the role of lipids in membranes. Chem Phys Lipids 40:127-144

Davis JH, Bloom M, Butler KW, Smith ICP (1980) The temperature dependence of molecular order and the influence of cholesterol in Archoleplasma laidlawii membranes. Biochim Biophys Acta 597:477-491

De Planque MRR, Killian JA (2003) Protein-lipid interactions studied with designed transmembrane peptides: role of hydrophobic matching and interfacial anchoring. Mol Membr Biol 20:271284

Doux JPF, Killian JA (2010) Do cation-pI interactions occur in lipid bilayers between phosphatidylcholine headgroups and interfacially localized tryptophans? Biophys J 98(3):486a. Suppl 1

Dufourc EJ, Parish EJ, Chitrakorn S, Smith ICP (1980) Structural and dynamical details of cholesterol-lipid interactions as revealed by deuterium NMR. Biochemistry 23:6062-6071

Dzikovski BG, Borbat PP, Freed JH (2004) Spin-labeled gramicidin A: channel formation and dissociation. Biophys J 87:3504-3517

Edholm O, Johansson J (1987) Lipid bilayer polypeptide interactions studied by molecular dynamics simulation. Eur Biophys J 14:203-209

Evans G, Pettifer RF (2001) Chooch: a program for deriving anomalous-scattering factors from $\mathrm{X}$-ray fluorescence spectra. J Appl Cryst 34:82-86

Fahsel S, Pospiech E-M, Zein M, Hazlet TL, Gratton E, Winter R (2002) Modulation of concentration fluctuations in phaseseparated lipid membranes by polypeptide insertion. Biophys $\mathbf{J}$ 83:334-344

Giewekemeyer K, Salditt T (2007) Counterion distribution near a monolayer of variable charge density. EPL 79:18003

Greenspan L (1977) Humidity fixed points of binary saturated aequeous solutions. J Res Natl Bureau Stan A Phys Chem 81A:89-96

Gullikson E (1995-2008) X-ray interactions with matter. Center for X-ray optics, Lawrence Berkeley National Laboratory, Berkeley

Harroun TA, Heller WT, Weiss TM, Yang L, Huang HW (1999) Experimental evidence for hydrophobic matching and membrane mediated interactions in lipid bilayers containing gramicidin. Biophys J 76:937-945

He K, Ludtke SJ, Wu Y, Huang HW (1993) X-ray scattering with momentum transfer in the plane of membrane-application to gramicidin organization. Biophys J 64:157-162

Hite RK, Gonen T, Harrison SC, Walz T (2008) Interaction of lipids with aquaporin-0 and other membrane proteins. Pflug Arch Eur J Physiol 456:651-661

Ho C, Slater SJ, Stubbs CD (1995) Hydration and order in lipid bilayers. Biochemistry 34:6188-6195

Husslein T, Moore PB, Zhong Q, Newns DM (1998) Molecular dynamics simulations of a hydrated diphytanol phosphatidylcholine lipid bilayer containing an aplpha-helical bundle of four transmembrane domains of the influenza A virus M2 protein. Faraday Discuss 111:201-208

Kelkar DA, Chattopadhyay A (2007) The gramicidin ion channel: a model membrane protein. Biochim Biophys Acta 1768:20112025

Khattari Z, Brotons G, Arbely A, Arkin IT, Metzger TH, Salditt T (2005) SARS E protein in phospholipid bilayers: an anomalous X-ray reflectivity study. Phys B 357:34-38

Khattari Z, Arkin IT, Salditt T (2006a) Viral ion channel proteins in model membranes: a comparative study by $\mathrm{X}$-ray reflectivity. Eur Biophys J 36:45-55

Khattari Z, Brotons G, Akkawi M, Arbely A, Arkin IT, Salditt T (2006b) SARS coronavirus E protein in phospholipid bilayers: a X-ray scattering study. Biophys J 90:2038-2050 
Killian JA, Nyholm TKM (2006) Peptides in lipid bilayers: the power of simple models. Curr Opin Struct Biol 16:473-479

Koeppe RE II, Andersen OS (1996) Engineering the gramicidin channel. Annu Rev Biophys Biomol Struct 25:231-258

Koert U, Al-Momani L, Pfeifer JR (2004) Synthetic ion channels. Synthesis 8:1129-1146

Krishnakumar SS, London E (2007) The control of transmembrane helix transverse position in membranes by hydrophilic residues. J Mol Biol 374:1251-1269

Küsel A, Khattari Z, Schneggenburger PE, Banerjee A, Salditt T, Diederichsen U (2007) Conformation and interaction of a D,Lalternating peptide with a bilayer membrane: X-ray reflectivity, CD, and FTIR spectroscopy. Chem Phys Chem 8:2336-2343

Lafleur M, Cullis PR, Bloom M (1990a) Modulation of the orientational order profile of the lipid acyl chain in the $L_{\alpha}$ phase. Eur Biophys J 19:55-62

Lafleur M, Cullis PR, Fine B, Bloom M (1990b) Comparison of the orientational order of lipid chains in the $L_{\alpha}$ and the $H_{\text {II }}$ phase. Biochemistry 29:8325-8333

Laguie P, Zuckermann MJ, Roux B (1998) Protein inclusion in lipid membranes: a theory based on the hypernetted chain integral equation. Faraday Discuss 111:165-172

Lagüe P, Zuckermann MJ, Roux B (2001) Lipid-mediated interactions between intrinsic membrane proteins: dependence on protein size and lipid composition. Biophys J 81:276-284

Li C, Salditt T (2006) Structure of magainin and alamethicin in model membranes studied by X-ray reflectivity. Biophys J 91:32853300

Long RA, Hruska F, Gesser HD, Hsia JC, Williams R (1970) Membrane condensing effect of cholesterol and the role of its hydroxyl group. Biochem Biophys Res Commun 41:321-327

Marčelja S (1974) Chain ordering in liquid crytals II. Structure of bilayer membranes. Biochim Biophys Acta 367:165-176

Marčelja S (1976) Lipid-mediated protein interaction in membranes. Biochim Biophys Acta 455:1-7

Marsh D (1996) Peptide models for membrane channels. Biochem J 315:345-361

Marsh D, Horváth L (1998) Structure, dynamics and composition of the lipid-protein interface. Perspectives from spin-labeling. Biochim Biophys Acta 1376:267-296

Mouritsen OG, Zuckermann MJ (2004) What's so special about cholesterol? Lipids 39:1101-1113

Münster C, Lu J, Schinzel S, Bechinger B, Salditt T (2000) Grazing incidence X-ray diffraction of highly aligned phospholipid membranes containing the antimicrobial peptide magainin 2 . Eur Biophys J 28:683-688

Naik VM, Krimm S (1986a) Vibrational analysis of the structure of gramicidin A-I. Normal mode analysis. Biophys J 49:11311145

Naik VM, Krimm S (1986b) Vibrational analysis of the structure of gramicidin A-II. Vibrational spectra. Biophys J 49:1147-1154

Oldfield E, Chapman D (1972) Dynamics of lipids in membranes: heterogeneity and the role of cholesterol. FEBS Lett 23:285-297

Oldfield E, Meadows M, Rice D (1978) Spectroscopic studies of specifically deuterium labeled membrane systems. Nuclear magnetic resonance investigation of the effects of cholesterol on model systems. Biochemistry 17:2727-2740

Palsdottir H, Hunte C (2004) Lipids in membrane protein structures. Biochim Biophys Acta 1666:2-18

Perly B, Smith ICP, Jarell HC (1985) Effects of the replacement of a double bond by a cyclopropane ring in phosphatidylethanolamines: a ${ }^{2} \mathrm{H}-\mathrm{NMR}$ study of the phase transition and molecular organization. Biochemistry 24:1055-1063

Qian S, Wang W, Yang L, Huang HW (2008) Structure of alamethicin pore reconstructed by X-ray diffraction analysis. Biophys J 94:3512-3522
Reusch T (2009) Nichtlamellare Strukturen in Lipidmembranstapeln in Abhängigkeit der Hydratisation: Probenumgebung, Phasendiagramme und Bestimmung der Elektronendichte. Diploma Thesis, Georg-August-Universität Göttingen, Göttingen

Ridder A, Skupjen P, Unterreitmeier S, Langosch D (2005) Tryptophan supports interaction of transmembrane helices. J Mol Biol 354:894-902

Salditt T, Li C, Spaar A, Mennicke U (2002) X-ray reflectivity of solid-supported multilamellar membranes. Eur Phys J E Soft Matter Biol Phys 7:105-116

Salditt T, Li C, Spaar A (2006) Structure of antimicrobial peptides and lipid membranes probed by interface-sensitive X-ray scattering. Biochim Biophys Acta 1758:1483-1498

Schneggenburger PE (2010) Synthesis, organization and structural studies of peptide motifs interacting with membrane systems. $\mathrm{PhD}$ Thesis, 1 edn. Book on Demand $\mathrm{GmbH}$, Norderstedt

Schneggenburger PE, Beerlink A, Worbs B, Salditt T, Diederichsen U (2009) A novel heavy-atom label for side-specific peptide iodination: synthesis, membrane incorporation and X-ray reflectivity. Chem Phys Chem 10:1567-1576

Schneggenburger PE, Müllar S, Worbs B, Steinem C, Diederichsen U (2010) Molecular recognition at the membrane-water interface: controlling integral peptide helices via off-membrane nucleobase pairing. J Am Chem Soc 132:8020-8028

Seelig A, Seelig J (1977) Effect of a single cis bond on the structure of a phospholipid bilayer. Biochemistry 16:45-50

Seul M, Sammon MJ (1990) Preparation of surfactant multilayer films on solid substrates by deposition from organic solution. Thin Solid Films 185:287-289

Spaar A, Salditt T (2003) Short range order of hydrocarbon chains in fluid phospholipid bilayers studied by X-ray diffraction from highly aligned oriented membranes. Biophys J 85:1576-1584

Spaar A, Münster C, Salditt T (2004) Conformation of peptides in lipid membranes studied by $\mathrm{X}$-ray grazing incidence scattering. Biophys J 87:396-407

Sternin E, Fine B, Bloom M, Tilcock CPS, Wong KF, Cullis PR (1988) Acyl chain orientational order in the hexagonal $H_{\text {II }}$ phase of phospholipid-water dispersions. Biophys J 54:689-694

Stockton GW, Smith ICP (1976) A deuterium nuclear magnetic resonance study of the condensing effect of cholesterol on egg phosphatidylcholine bilayer membranes. Chem Phys Lipids 17:251-263

Strandberg E, Ulrich AS (2004) NMR methods for studying membrane-active antimicrobial peptides. Concepts Magn Reson $23 \mathrm{~A}: 89-120$

Tamm L, Tatulian SA (1997) Infrared spectroscopy of proteins and peptides in lipid bilayers. Q Rev Biophys 30:365-429

Träuble H, Overath P (1973) The structure of Escherichia coli membranes studied by fluorescence measurements of lipid phase transitions. Biochim Biophys Acta 307:491-512

Weinhausen B (2010) Strukturanalyse von Phospholipidmembranen in Abhängigkeit von der Hydratisation und dem Cholesteringehalt: Elektronendichteprofile und Acylketten. Diploma Thesis, Georg-August-Universität Göttingen, Göttingen

Wimley WC, White SH (2000) Designing transmembrane $\alpha$-helices that insert spontaneously. Biochemistry 39:4432-4442

Winston PW, Bates DH (1960) Saturated solutions for the control of humidity in biological research. Ecology 41:232-237

Wu Y, He K, Ludtke SJ, Huang HW (1995) X-ray diffraction study of lipid bilayer membrane interacting with amphiphilic helical peptides: diphytanoyl phosphatidylcholine with alamethicin at low concentrations. Biophys J 68:2361-2369

Wu Y, Huang HW, Olah GA (1990) Method of oriented circular dichroism. Biophys J 57:797-806 